\title{
Repurposing Pomalidomide as a Neuroprotective Drug: Efficacy in an Alpha-Synuclein-Based Model of Parkinson's Disease
}

\author{
Maria Francesca Palmas ${ }^{1}$ Anna Ena ${ }^{1}$. Chiara Burgaletto ${ }^{2} \cdot$ Maria Antonietta Casu $^{3} \cdot$ Giuseppina Cantarella $^{2}$. \\ Ezio Carboni ${ }^{1} \cdot$ Michela Etzi ${ }^{1}$. Alfonso De Simone ${ }^{4}$. Giuliana Fusco ${ }^{5}$. Maria Cristina Cardia ${ }^{6}$. Francesco Lai $^{6}$. \\ Luca Picci $^{6}$. David Tweedie ${ }^{7}$ - Michael T. Scerba ${ }^{7} \cdot$ Valentina Coroneo $^{8} \cdot$ Renato Bernardini $^{2} \cdot$ Nigel H. Greig $^{7}$. \\ Augusta Pisanu $^{9} \cdot$ Anna R. Carta ${ }^{1}[$
}

Accepted: 24 December 2021 / Published online: 24 January 2022

(c) The Author(s) 2022

\begin{abstract}
Marketed drugs for Parkinson's disease (PD) treat disease motor symptoms but are ineffective in stopping or slowing disease progression. In the quest of novel pharmacological approaches that may target disease progression, drug-repurposing provides a strategy to accelerate the preclinical and clinical testing of drugs already approved for other medical indications. Here, we targeted the inflammatory component of PD pathology, by testing for the first time the disease-modifying properties of the immunomodulatory imide drug (IMiD) pomalidomide in a translational rat model of PD neuropathology based on the intranigral bilateral infusion of toxic preformed oligomers of human $\alpha$-synuclein $(\mathrm{H}-\alpha \mathrm{SynOs})$. The neuroprotective effect of pomalidomide $(20 \mathrm{mg} / \mathrm{kg}$; i.p. three times/week $48 \mathrm{~h}$ apart) was tested in the first stage of disease progression by means of a chronic two-month administration, starting 1 month after H- $\alpha$ SynOs infusion, when an already ongoing neuroinflammation is observed. The intracerebral infusion of $\mathrm{H}-\alpha \mathrm{SynO}$ induced an impairment in motor and coordination performance that was fully rescued by pomalidomide, as assessed via a battery of motor tests three months after infusion. Moreover, $\mathrm{H}-\alpha$ SynOs-infused rats displayed a $40-45 \%$ cell loss within the bilateral substantia nigra, as measured by stereological counting of $\mathrm{TH}+$ and Nissl-stained neurons, that was largely abolished by pomalidomide. The inflammatory response to $\mathrm{H}-\alpha$ SynOs infusion and the pomalidomide treatment was evaluated both in CNS affected areas and peripherally in the serum. A reactive microgliosis, measured as the volume occupied by the microglial marker Iba-1, was present in the substantia nigra three months after $\mathrm{H}-\alpha \mathrm{SynO}$ infusion as well as after $\mathrm{H}-\alpha \mathrm{SynO}$ s plus pomalidomide treatment. However, microglia differed for their phenotype among experimental groups. After $\mathrm{H}-\alpha$ SynOs infusion, microglia displayed a proinflammatory profile, producing a large amount of the proinflammatory cytokine TNF- $\alpha$. In contrast, pomalidomide inhibited the TNF- $\alpha$ overproduction and elevated the anti-inflammatory cytokine IL-10. Moreover, the H- $\alpha$ SynOs infusion induced a systemic inflammation with overproduction of serum proinflammatory cytokines and chemokines, that was largely mitigated by pomalidomide. Results provide evidence of the disease modifying potential of pomalidomide in a neuropathological rodent model of PD and support the repurposing of this drug for clinical testing in PD patients.
\end{abstract}

Keywords Immunomodulation · Alpha-synuclein $\cdot$ Cytokine $\cdot$ Neuroprotection $\cdot$ Motor impairment $\cdot$ Drug repositioning

\section{Introduction}

Despite massive preclinical and clinical effort, marketed drugs for the treatment of PD remain symptomatic in their efficacy and appear ineffective in stopping or slowing

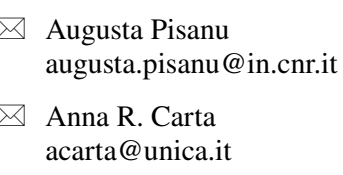

Augusta Pisanu

augusta.pisanu@in.cnr.it

$\triangle$ Anna R. Carta acarta@unica.it

Extended author information available on the last page of the article disease progression. An increasing knowledge of PD neuropathology together with the wide availability of drugs with regulatory-approved safety data, endorses drug-repurposing as an appealing strategy to accelerate the preclinical and clinical testing of drugs already approved for other medical indications [1-4]. In this quest, drugs targeting components of the inflammatory response represent an attractive diseasemodifying strategy, and several clinically available immunosuppressive and immunomodulatory drugs have been tested for their neuroprotective activity in preclinical models of PD [5]. Chronic neuroinflammation is a recognized 
neuropathological trait of PD, originating from dysfunctional glial cells in the brain, likely driven by pathological interactions with toxic $\alpha$-synuclein ( $\alpha$-Syn) [6, 7]. Glial cells in PD lose their homeostatic and defense function in favor of a pathological gain of toxic functions. This results in an unremittent production of proinflammatory molecules, such as tumor necrosis factor (TNF)- $\alpha$, within degenerating mesencephalic areas [8-12]. In addition, analyses of serum and peripheral organs of PD patients have revealed a dysregulated cytokine content $[13,14]$, suggesting that the inflammatory pathology can involve the whole organism [15].

An attractive class of drugs in this context are the immunomodulatory imide drugs (IMiDs), such as thalidomide and its analogs, that have shown beneficial effects on neurodegeneration in preclinical models of PD [1, 16, 17]. Compared to drugs with immunosuppressive activity, whose clinical translation is often hampered by toxicological and pharmacokinetic limitations, IMiDs offer several advantageous features that make them more suitable for treating chronic neurological disorders. Most importantly, both in silico and in vivo data have shown that IMiDs cross the blood-brain barrier (BBB) and readily enter the brain from plasma [18, 19] (Cardia et al. submitted). IMiDs display a potent anti-inflammatory effect, acting primarily through the inhibition of TNF- $\alpha$ production via post-translational mechanisms, and consequent dampening of the inflammatory cascade [20-24]. Among FDA-approved IMiDs, pomalidomide is a regulatory-approved drug used in the oncology area for the treatment of specific types of cancer such as relapsed/refractory multiple myeloma and Kaposi sarcoma. Pomalidomide is particularly attractive because it displays a TNF- $\alpha$ inhibitory action of up to 50,000-fold greater than the parent compound thalidomide $[25,26]$, and it has a favorable BBB permeability in rodents [27] (Cardia et al. submitted). In a recent study, we have reported the neuroprotective efficacy of pomalidomide in the Drosophila LRRK2 $2^{\text {WD40 }}$ genetic model of PD, where we have shown that pomalidomide rescued the motor activity and attenuated the dopaminergic neuron damage and degeneration [16]. Moreover, as compared to thalidomide and derivatives, pomalidomide displays less adverse effects related to neurotoxic activity $[25,28]$.

Here, we tested the disease-modifying properties of pomalidomide, including the central and peripheral drug effects on inflammatory markers, in the $\alpha$-Syn-based rodent model of PD obtained by the central bilateral infusion of toxic oligomers of $\alpha$-Syn ( $\alpha$ SynOs) into the rat substantia nigra pars compacta (SNpc) [29, 30]. Despite an increasing literature suggesting a neuroprotective property of IMiDs in PD, most studies have been designed in toxin-based models, whereas data from the $\alpha$-Syn-based translational model remain scarce or totally absent [17]. Yet, the central neuropathological role of $\alpha$-Syn is widely acknowledged, and modelling the $\alpha$-Syn neuropathology of PD offers a valuable tool to investigate novel neuroprotective therapies, when compared with classical toxin-based models, to further bridge the gap between preclinical and clinical studies $[31,32]$. In this light, there is an urgent need for additional studies in these translational models of PD to better define the efficacy of IMiDs that may foster the translation to their clinical testing.

In order to comprehensively assess the disease-modifying properties of pomalidomide we chronically treated $\alpha$ SynOsinfused rats with the drug and evaluated their motor performance and coordination ability with a battery of highly sensitive tests. Thereafter, to assess the neuroprotective activity of pomalidomide we analyzed stereologically the number of tyrosine hydroxylase $(\mathrm{TH})^{+}$cells within the SNpc. The inflammatory response induced by $\alpha$ SynOs infusion and the pomalidomide activity toward this pathological trait, was appraised both in CNS affected areas by immunofluorescence analysis of the phenotype of microglia, defined as Iba$1^{+}$(ionized calcium-binding adapter molecule 1 ) cells, and peripherally in the serum by multiplex ELISA for cytokines and chemokines.

\section{Materials and Methods}

All procedures were performed in accord with the ARRIVE guidelines and in accordance with the guidelines and protocols approved by the European Community (2010/63UE L 276 20/10/2010). Experimental protocols were approved by the Italian Ministry of Health (authorization N 766/2020PR). All efforts were made to minimize animal pain and discomfort and to reduce the number of experimental animals used.

\section{Production of Recombinant H-aSyn}

Recombinant human $\alpha$ Syn (H- $\alpha$ Syn) was purified in E. coli using plasmid pT7-7 encoding for the protein as previously described [30]. The expression was induced with $1 \mathrm{mM}$ IPTG at $37{ }^{\circ} \mathrm{C}$ for $4 \mathrm{~h}$. The cell lysate was centrifuged at 22,000 $\mathrm{g}$ (Beckman Coulter, Brea, USA) for $30 \mathrm{~min}$, and the supernatant was then heated for $20 \mathrm{~min}$ at $70{ }^{\circ} \mathrm{C}$. After centrifugation at $22,000 \mathrm{~g}$, two steps of precipitation and centrifugation were employed and, in particular, $10 \mathrm{mg} \cdot \mathrm{mL}^{-1}$ streptomycin sulfate was added to the supernatant for DNA precipitation. Subsequently, $360 \mathrm{mg} \cdot \mathrm{mL}^{-1}$ ammonium sulfate was added to the supernatant to precipitate the recombinant $\mathrm{H}-\alpha \mathrm{Syn}$. The obtained pellet was resuspended in $25 \mathrm{mM}$ Tris- $\mathrm{HCl}, \mathrm{pH} 7.7$ and, after dialysis against the same buffer, loaded onto an anion exchange column (26/10 Q sepharose high performance, GE Healthcare, Little Chalfont, $\mathrm{UK}$ ) to be eluted with a $0-1 \mathrm{M} \mathrm{NaCl}$ step gradient. 
Further purification was achieved by applying size exclusion chromatography (Hiload 26/60 Superdex 75 preparation grade, GE Healthcare). The purity of the sample was analyzed by SDS-PAGE, and the protein concentration was determined from the absorbance at $275 \mathrm{~nm}$ using an extinction coefficient of $5600 \mathrm{M}^{-1} \cdot \mathrm{cm}^{-1}$.

\section{Purification of $\mathrm{H}$-asynO}

Toxic oligomeric samples were prepared from purified recombinant $\mathrm{H}-\alpha \mathrm{Syn}$ as previously described $[29,30]$. Lyophilized protein was resuspended in PBS buffer at a $\mathrm{pH}$ of 7.4 and a concentration of $12 \mathrm{mg} \cdot \mathrm{mL}^{-1}$, then passed through a $0.22 \mu \mathrm{m}$ cutoff filter before incubation at $37{ }^{\circ} \mathrm{C}$ for $24 \mathrm{~h}$ without agitation. Residual fibrillar species were removed by ultracentrifugation for $1 \mathrm{~h}$ at $288,000 \mathrm{~g}$, and excess of monomers were removed using several filtration steps with $100 \mathrm{kDa}$ cutoff membranes. Samples of the toxic $\mathrm{H}-\alpha$ Syn oligomers prepared in this manner are stable for many days at room temperature, but in this study were used within 2 days of their production. At the end of the purification procedure, and prior to intracerebral inoculation, oligomers were tested for endotoxin contamination via the LAL (Limulus Amebocyte Lysate) assay (Kairosafe, Italy).

\section{Animals, Stereotaxic Surgery, and Pharmacological Treatment}

Male Sprague-Dawley rats (275-300 g, Envigo) were housed in groups of three to four in standard conditions of temperature $\left(21 \pm 1{ }^{\circ} \mathrm{C}\right)$ and humidity (60\%) under a $12 \mathrm{~h}$ light/dark cycle (lights on 7:00 A.M) with standard chow and water available ad libitum.
Forty-five rats were deeply anesthetized with Fentanyl $(3 \mathrm{mg} / \mathrm{kg})$ and medetomidine hydrochloride $(0.35 \mathrm{mg} /$ $\mathrm{kg}$ ) and were stereotaxically injected with $5 \mu \mathrm{L}$ of human $\alpha \mathrm{SynO}(\mathrm{H}-\alpha \mathrm{SynO}$ ) into the $\mathrm{SNpc}$ (coordinates relative to bregma; $-5.4 \mathrm{~mm}$ anteroposterior; $\pm 1.9 \mathrm{~mm}$ from the midline; $-7.2 \mathrm{~mm}$ beneath the dura) bilaterally at the rate of 1 $\mu \mathrm{L} / \mathrm{min}$ via a silica microinjector and according to the atlas of Paxinos and Watson [33], as previously described [29]. Control animals received sterile PBS (Fig. 1).

One-month post-surgery rats were chronically administered with pomalidomide (Pom, $20 \mathrm{mg} / \mathrm{kg}$; i.p.) or saline on alternate days 3 days/week for 2 months and sacrificed $24 \mathrm{~h}$ after the last injection. The 1-month interval after infusion was chosen based on a previous observation [29] of neuronal damage and brain inflammation 30 days after oligomer infusion, which can be considered as a prodromal stage of PD, evolving into neuronal loss at three months post-infusion. Pomalidomide was nanosuspended in an aqueous solution of Tween $80(0.75 \%)$ using the Wet media milling technique, as described [34] (Cardia et al. submitted), in order to optimize its bioavailability after i.p. injection. The experimental groups were as follows: (i) vehicle + saline (Veh-Sal); (ii) $\mathrm{H}-\alpha \mathrm{SynOs}+$ saline (H- $\alpha$ SynOs-Sal); (iii) Veh + Pom (VehPom); (iv) $\mathrm{H}-\alpha \mathrm{SynOs}+\mathrm{Pom}$ (H- $\alpha$ SynOs-Pom).

\section{Behavioral Tests}

A range of behavioral studies were conducted over a 10-day interval, beginning 12 weeks after $\mathrm{H}-\alpha$ SynOs infusion. Prior to each test, rats were acclimated to the testing room for $30 \mathrm{~min}$ in order to avoid any alteration in behavioral parameters induced by the novel environment. Tests were carried out between 9 am and $3 \mathrm{pm}$. All tests were performed and analyzed by individuals blinded to the experimental conditions.

Experimental groups:

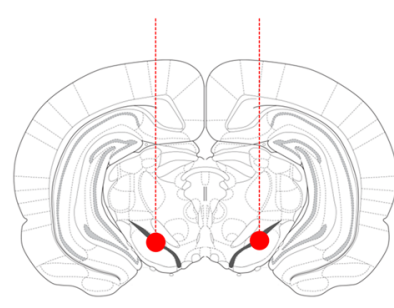

Vehicle-Saline; Vehicle-Pomalidomide; H-aSynOs-Saline; H-aSynOs-Pomalidomide

\begin{tabular}{lcr}
\hline \multicolumn{1}{c}{1 month p.i. } & T months p.i. \\
Bilateral H- $\alpha$ SynOs infusion & Pomalidomide treatment & Motor performance; \\
$(5 \mu \mathrm{l} ; 0.5 \mathrm{mg} / \mathrm{ml}$ per side). & (i.p., $20 \mathrm{mg} / \mathrm{kg}, 3 \mathrm{t} / \mathrm{w})$ & brain and blood collection for: \\
3 months SD rats & & Immunostaining; Multiplex ELISA
\end{tabular}

Fig. 1 Experimental timeline showing the site for $\mathrm{H}-\alpha \mathrm{SynOs}$ infusion in vivo and the experimental protocol 


\section{Challenging Beam Walk Test}

The challenging beam test was used to assess motor coordination and balance as shown previously [29] with a protocol adapted from Drucker-Colín and García-Hernández [35], Fleming et al. [36], and Korecka et al. [37]. The testing apparatus consisted of a $2 \mathrm{~m}$ wooden beam placed between a starting platform, elevated $40 \mathrm{~cm}$ from the floor, and the home cage, with a slope of $15^{\circ}$. Three different beam widths were used: 15,10 , and $5 \mathrm{~mm}$. All rats were trained to run across the different beams for 3 days before initiation of testing and, on the test day, they were videotaped. Briefly, each rat was placed at the lower end of the beam and the number of stepping errors was counted while traversing the beam to reach the home cage. The same procedure was repeated for the three different beam widths [29]. Some animal failed to complete the task within the established time-frame or fell off the beam, reflecting a sensorimotor impairment as previously reported [35]. In these events, in order to assign a numeric score to the impairment the error score was increased by adding a numerical increment based on the following criteria: (i) 0.25 increment, when the animal completed $75 \%$ of the beam; (ii) 0.5 increment, when the animal completed $50 \%$ of the beam; (iii) 0.75 when it only completed $25 \%$ of the beam.

\section{Vermicelli Handling Test}

The vermicelli handling test was used as a measure of forepaw ability [38, 39]. During the week prior to testing, all rats were exposed to the pasta pieces several times in order to avoid any neophobic responses. On the test day, each rat was placed in a box with dark walls, with a mirror set $45^{\circ}$ below it, and exposed to three $7 \mathrm{~cm}$ uncooked vermicelli strands. Each trial was videotaped for later analysis. Trials were invalidated should the rat break the pasta piece during eating or if less than $90 \%$ of the recorded eating session showed a clear view of the paws/digits $[39,40]$. The primary outcome measures for this test were as follows: (a) number of forepaw adjustments per trial, defined as any distinct removal and replacement of the paw on the pasta piece; (b) frequency of atypical handling patterns. These atypical behaviors included the following: (1) paws together when long-paws placed symmetrically when the piece of pasta was $3.5 \mathrm{~cm}$ or greater in length; (2) guide and grasp switch - the roles of the guide limb and grasp limb are switched during eating; (3) failure to contact - the paw does not contact the pasta piece during eating; (4) drop-the pasta piece is dropped after eating is initiated; (5) paws apart when short-paws placed asymmetrically when the pasta piece is short [38]. These measures were scored 1 if exhibited and 0 when not, and their frequency was then summed.

\section{Gait Test}

A gait test was run on an apparatus consisting of an arena placed on a base with a transparent floor and a mirror mounted inside. The arena was $15 \mathrm{~cm}$ wide, $25 \mathrm{~cm}$ tall, and $148 \mathrm{~cm}$ long. Walls and lid were made of black alveolar polypropylene panels to provide high contrast for white rodents. The base was a wooden frame $(24 \times 24 \times 160 \mathrm{~cm})$ provided with a plexiglass top, which served as a floor for the overlying arena. A mirror was housed inside and set $45^{\circ}$ below the arena. Animals were acclimated to the arena for several minutes before testing. For testing, each rat independently and voluntarily explored the arena at self-selected velocities, and three runs across the arena were videotaped at $120 \mathrm{fps}$. For analysis, only the central portion of the arena was considered (100 cm long), whereas the starting and the terminal portions $(24 \mathrm{~cm}$ each) were not considered. The time spent and the number of steps made to cross the central part of the arena were measured for each rat. Moreover, strides were analyzed by measuring the distance between paw placements for every step, on a stack of calibrated frames on IMAGE J (https://imagej.nih.gov/ij/). For each, subject data are expressed as averaged velocity $(\mathrm{cm} / \mathrm{s})$, steps per time unit $(\mathrm{n} /)$, and averaged stride length $(\mathrm{cm})$.

\section{Immunohistochemistry}

After the behavioral testing and within $24 \mathrm{~h}$ of their last injection, rats were anesthetized and transcardially perfused with ice-cold 0.1 M PBS (pH 7.4) followed by $4 \%$ buffered paraformaldehyde. After perfusion, the brain was carefully removed, post-fixed overnight in $4 \%$ paraformaldehydePBS and stored in $0.1 \% \mathrm{NaN}_{3}$-PBS at $4{ }^{\circ} \mathrm{C}$. Thereafter, $40-\mu \mathrm{m}$-thick serial sections of midbrain and striatum were vibratome-cut [41].

For stereological quantification, midbrain sections were pre-incubated in normal donkey serum and then immunoreacted with polyclonal rabbit anti-TH (1:1000, Millipore, Burlington, MA, USA) primary antibody. The reaction was amplified using a biotinylated secondary antibody and visualized by the classic avidin-peroxidase complex (ABC, Vector, UK) protocol, using 3,30-diaminobenzidine (SigmaAldrich, St. Louis, MO, USA) as a chromogen. Sections were then counterstained with cresyl violet.

For immunofluorescence, midbrain sections were preincubated with a blocking solution with normal serum/BSA and were then immunoreacted with the following unconjugated primary antibodies for double immunolabeling: goat polyclonal anti Iba-1 (1:1000; Novus Biologicals, Littleton, CO, USA); rabbit polyclonal anti TNF- $\alpha$ (1:500, Novus Biologicals, Littleton, CO, USA); rabbit polyclonal anti IL-10 (1:200, Abbiotec, Escondido, CA, USA); mouse monoclonal 
anti CD3 (1:50, Santa Cruz Biotechnology, Santa Cruz, CA, USA). For fluorescence visualization of Iba-1 and CD3 a two-step indirect labelling protocol was used, while a threestep detection was performed to increase the signal of TNF- $\alpha$ and IL-10 by combining biotin-SP-conjugated IgG (1:500, Jackson Immunoresearch, West Grove, PA, USA) and streptavidin-fluorescein (1:400, Jackson Immunoresearch, West Grove, PA, USA), as previously described [29]. Images were acquired using a spinning disk confocal microscope (Crisel Instruments, Rome, Italy) with a $\times 63$ magnification.

\section{Stereological Counting of TH Immunoreactivity}

All immunohistochemical reactions were analyzed by an operator blinded to the experimental groups, and different from the experimenter who performed the behavioral tests and histology. TH-immunoreactive neurons or Nissl-stained cells were counted bilaterally in the $\mathrm{SNpc}$, as previously described [42]. A dedicated software was used (Stereologer, System Planning and Analysis, Inc., Alexandria, VA, USA), linked to a motorized stage on a BX-60 Olympus light microscope (Olympus, Segrate, Italy). The total number of TH-stained cells was estimated by means of the optical fractionator method, which combines the optical dissector with the fractionator sampling scheme, giving a direct estimation of the number of 3-D objects unbiased by shape, size, and orientation [43]. A systematic random sampling of cells within the area of interest was achieved by "Stereologer" software. Equidistant counting frames (frame area $=50$ $\mu \mathrm{m}^{2}$ ) were obtained. Sampling fraction was delimited at low power and cells were sampled with $\mathrm{a} \times 40$ oil immersion objective through a defined depth with a $2 \mu \mathrm{m}$ guard zone. The coefficient of error (CE) for each estimation and animal ranged from 0.05 to 0.1 .

\section{Microscopy Analysis}

Qualitative and quantitative analyses for Iba-1, TNF- $\alpha$, IL-10, and $\mathrm{CD}^{+}{ }^{+}$were performed using a spinning disk confocal microscope (Crisel Instruments, Rome, Italy) with a $\times 63$ magnification. Surface rendering, colocalization, maximum intensity, and simulated fluorescence process algorithms were used (ImageJ and Imaris 7.3). To determine the Iba- 1 occupied volume, a stack was obtained from each dataset (40 images). In the resulting stacks, 10 regions of interest for the SNpc $(x=700 \mu \mathrm{m} ; y=700 \mu \mathrm{m}$; $z=40 \mu \mathrm{m}$ ) in each acquired section and for each animal were randomly chosen, and the volume of the elements calculated. For colocalization analysis, a colocalization channel was automatically generated by Imaris 7.3. In the resulting stacks each Iba- $1^{+}$cell was identified and selected, and the volume of the colocalized cytokine was calculated. Mean colocalization values obtained from cells analyzed in each animal from each experimental group $(n=6)$ were plotted as a frequency distribution displaying the percentage of colocalization between the selected cytokine signal and the selected Iba- $1^{+}$microglial cell. Histogram inspection indicated a different cytokine expression across experimental groups in a cell subpopulation, whereas a large population of cells maintained colocalization values similar to the vehicle (Fig. 6). For this reason, a deconvolution analysis was applied to the histograms in order to unmask subpopulations of cells affected by $\mathrm{H}-\alpha \mathrm{SynO}$ infusion and/or pomalidomide treatment. Based on the deconvolution results, an appropriate cut-off value was set in order to categorize the identified cell populations into high and low expressing cells; mean values within each class were calculated for each experimental group and statistically compared.

\section{Cytokine and Chemokine Analysis by Multiplex ELISA}

Serum samples were assayed using the Cytokine \& Chemokine 22-Plex Rat ProcartaPlexTM Panel (EPX220-30,122-901, Thermo Fisher Scientific, Vienna, Austria), according to the manufacturer's instructions. The concentrations of cytokines and chemokines were detected with the Luminex MAGPIX instrument (Luminex Corporation, Austin, TX) and data were analyzed with XPONENT® software (Luminex Corporation, Austin, TX). Any analyte with a concentration outside the linear range was excluded from the analysis.

\section{Statistical Analysis}

Outcome measures were evaluated by observers blinded to experimental conditions. Results are presented as mean \pm SEM, using Statistica 8 (Stat Soft Inc., Tulsa, OK, USA). Behavioral data were analyzed by two-way analysis of variance (ANOVA) with intranigral infusion and pharmacological treatment as factors, followed by Tukey's post hoc test, or by $t$-test where appropriate.

The results from the stereological analysis were statistically analyzed with a two-way analysis of variance (ANOVA) followed by Tukey's post hoc test, whereas the dataset of Iba-1 IR in the SNpc was analyzed by a KruskallWallis non-parametric test followed by Dunn's post hoc test.

Regarding the colocalization analysis of the cytokines, the effect of drug treatments on the identified subpopulation was determined by one-way ANOVA followed by Fisher's post hoc test for comparison between individual groups. Levels of serum cytokines and chemokines were 
statistically compared among the experimental groups by one-way ANOVA followed by Fisher's post hoc test.

For all the analyses, the level of significance was set at $p<0.05$.

\section{Results}

\section{Pomalidomide Mitigates H-aSynOs Infusion-Induced Deficits in Sensorimotor Function and Fine Motor Movement Execution}

To verify the functional outcome of pomalidomide-induced neuroprotection and specifically its efficacy against motor symptoms, a series of motor tests, including the challenging beam test, the vermicelli handling test and the gait test, were performed 3 months after $\mathrm{H}-\alpha$ SynOs infusion. In line with our previous study [29], H- $\alpha$ SynOs-infused rats developed a significant motor impairment in the beam walking test, expressed as the number of errors per step on the $10 \mathrm{~mm}$ and $5 \mathrm{~mm}$ beams (Fig. 2a, a1, a2), which was significantly higher than in control rats (Veh-Sal group). Moreover, $\mathrm{H}-\alpha$ SynOs-infused rats displayed an impairment in the gait test, expressed as a decrease of velocity, steps/second and stride length (Fig. 2b, b1, b2). Remarkably, pomalidomide treatment fully mitigated these sensorimotor impairments. In the beam test, pomalidomide-treated rats committed a similar number of stepping errors as the control group, which was significantly lower than $\mathrm{H}-\alpha$ SynOs-infused rats for the 10 and $5 \mathrm{~mm}$ width beams (Fig. 2a). Likewise, in the gait test, pomalidomide treatment significantly restored velocity, number of steps/second and stride length, as compared to $\mathrm{H}-\alpha$ SynOs-infused rats, to control values (Fig. $2 b, c, d$ ).

In the vermicelli handling test, $\mathrm{H}-\alpha \mathrm{SynO}$-infused rats showed fewer normal adjustments and an increased number of abnormal eating behaviors as compared with the Veh-Sal group (Fig. 2e, f), consistent with an impairment in fine movement execution. The administration of pomalidomide (H- $\alpha$ SynOs-Pom group) significantly increased the number of total adjustments during each trial, as compared to $\mathrm{H}-\alpha \mathrm{SynO} O$-infused rats, and decreased the number of abnormal eating behaviors, restoring these to control values and indicating a recovery from this motor impairment (Fig. 2e, f).

\section{Pomalidomide Protects Against Nigral Dopaminergic Degeneration in H-aSynOs-Infused Rats}

$\mathrm{H}-\alpha$ SynOs-infused rats displayed a $40-45 \%$ reduction, in comparison with control rats, both in the density and the
Fig. 2 Chronic treatment with pomalidomide significantly reduced motor impairments 3 months after $\mathrm{H}-\alpha$ SynOs infusion. Sensorimotor deficits were evaluated by the challenging beam walk test (a) and the gait test $(\mathbf{b}-\mathbf{d})$. Values represent the mean \pm SEM (two-way ANOVA and Tukey's post hoc test). ${ }^{\wedge} p<0.001 ;{ }^{*} p<0.01 ; * p<0.01$. Fine motor movements were assessed by the vermicelli handling test. Stacked bar charts show the number of normal adjustments (e) and the frequency of abnormal behaviors (f) made during the eating time. Values represent the mean \pm SEM. $* p<0.05$ vs Veh-Sal and $\mathrm{H}-\alpha$ SynOs-Pom (e); ${ }^{*} p<0.01$ vs Veh-Sal (f) by $t$-test

number of DA cells bilaterally in the right and left SNpc, as measured by stereological counting of $\mathrm{TH}^{+}$neurons (Fig. 3a-c). Remarkably, such a reduction in the number of $\mathrm{TH}^{+}$neurons was largely abolished when $\mathrm{H}-\alpha$ SynOs-infused rats were chronically treated for 2 months with pomalidomide, starting one month post-oligomer infusion (Fig. 3a-c), indicating that pomalidomide stopped the neurodegenerative process. To confirm that the changes observed in TH content reflected changes in cell number rather than a decline/ increase in enzyme levels, we stereologically counted Nisslcounterstained sections. Results were superimposable with our TH analysis, both in terms of density and number of cells, confirming that pomalidomide allayed $\mathrm{H}-\alpha$ SynOsinduced cell loss in the SNpc (Fig. 3d, e).

\section{Pomalidomide Effects on H-aSynOs-Induced Microgliosis in the SNpc}

Based on the pivotal role of neuroinflammation in PD neuropathology [8] and the expected primary mechanism of pomalidomide activity, we evaluated microglial reactivity within the SNpc by using Iba-1 as a marker of reactive microglia. Results, expressed as the total volume occupied by $\mathrm{Iba}-1^{+}$cells, are shown in Fig. $4 \mathrm{a}, \mathrm{b}$. Iba- $1^{+}$cell volume was similarly increased in the SNpc of $\mathrm{H}-\alpha \mathrm{SynOs}$-infused rats treated with either saline or pomalidomide, suggesting that the pharmacological treatment did not reduce the microgliosis.

\section{Pomalidomide Switches the Functional Phenotype of Microglia in the H-asynOs-Infused Rats}

To further investigate the immunomodulatory activity of pomalidomide within the CNS, and find a mechanistic correlate of neuroprotection, we characterized the functional phenotype of Iba- $1^{+}$cells by cytokine assessment. A colocalization analysis was performed for the pro-inflammatory cytokine TNF- $\alpha$ and the anti-inflammatory cytokine IL-10 within microglial cells. Our analysis of the data revealed a high variability in the content of both TNF- $\alpha$ and IL-10 within microglia in each experimental group, as shown by the non-normal distribution of the dataset, reflecting the high functional dynamicity of these cells. Therefore, to highlight 


\section{Challenging beam walk test}

a

$15 \mathrm{~mm}$

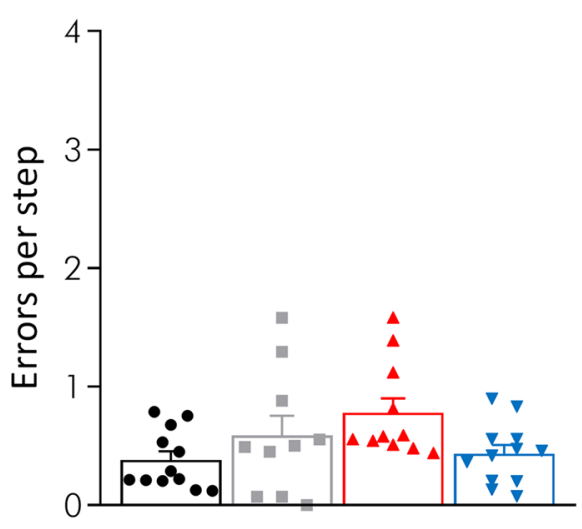

$10 \mathrm{~mm}$

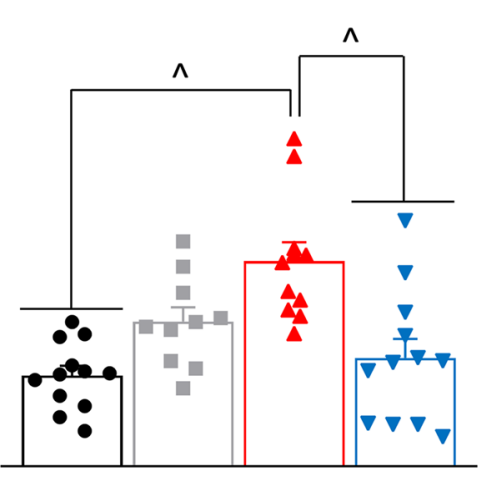

Gait test b

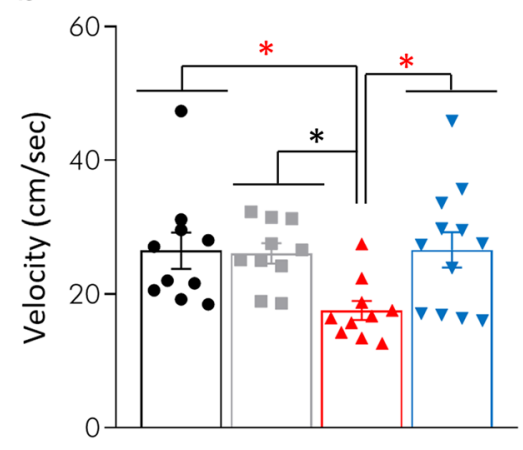

C

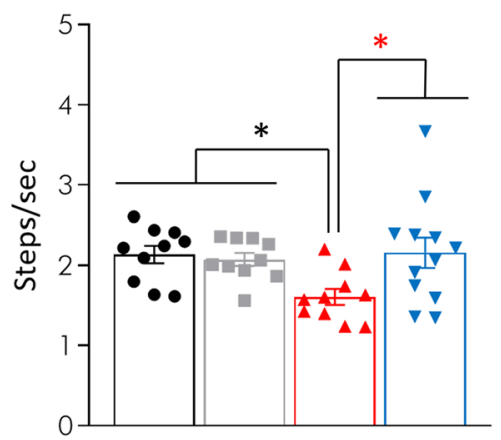

$5 \mathrm{~mm}$

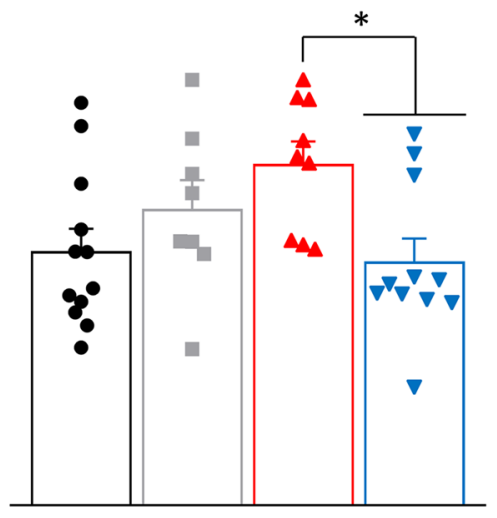

Vermicelli handling test

e

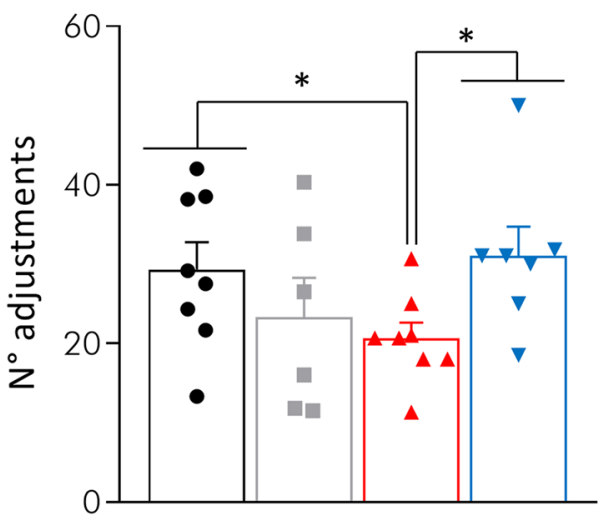

- Veh-Sal घ Veh-Pom d

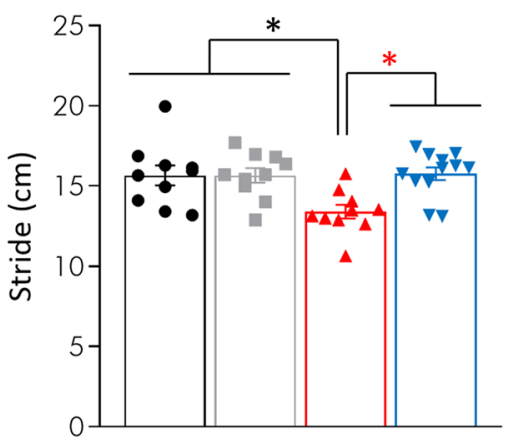

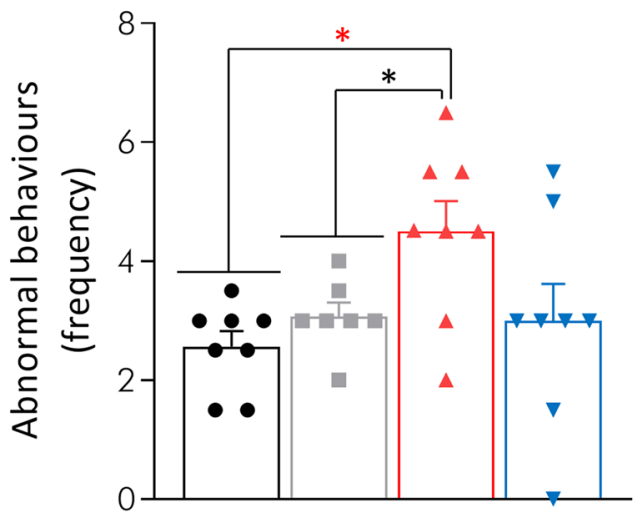

$\Delta$ h- $\alpha$ SynOs-Sal $\quad \nabla$ h- $\alpha$ SynOs-Pom 


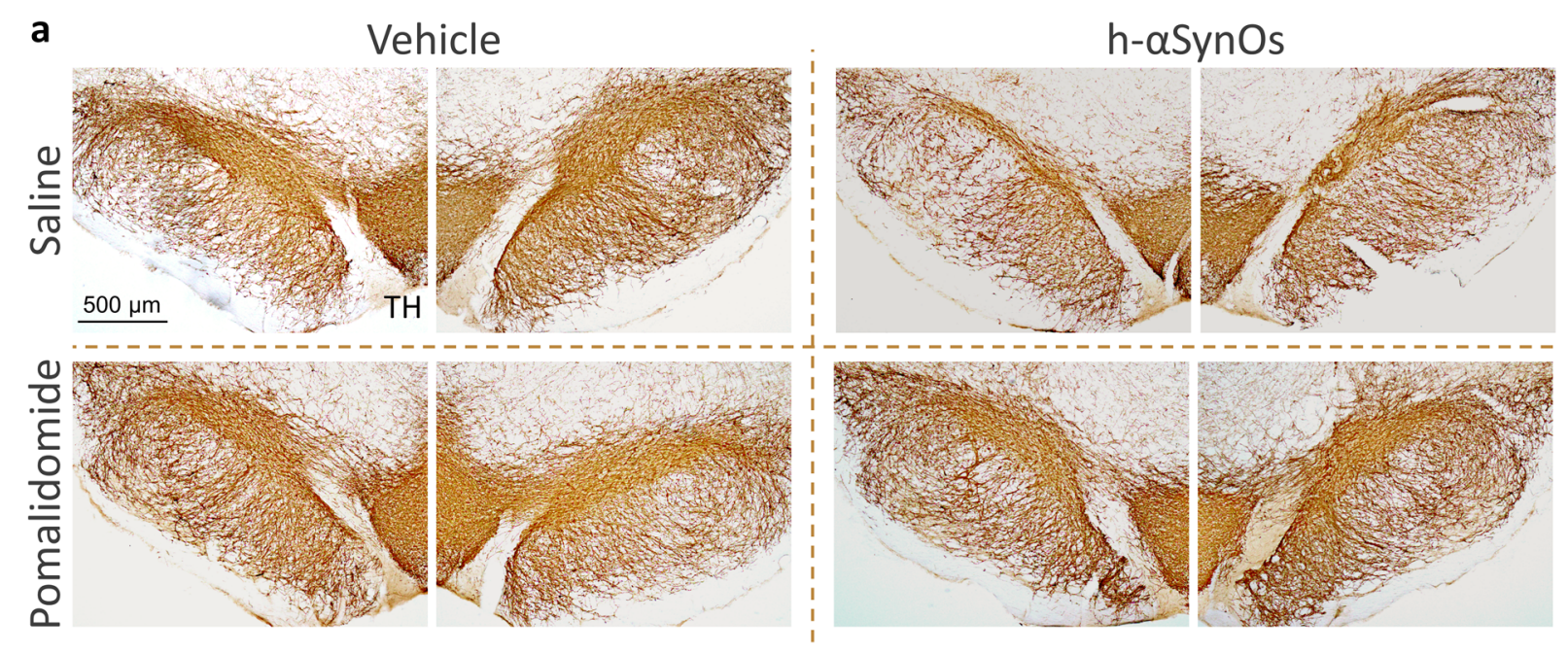

TH IR
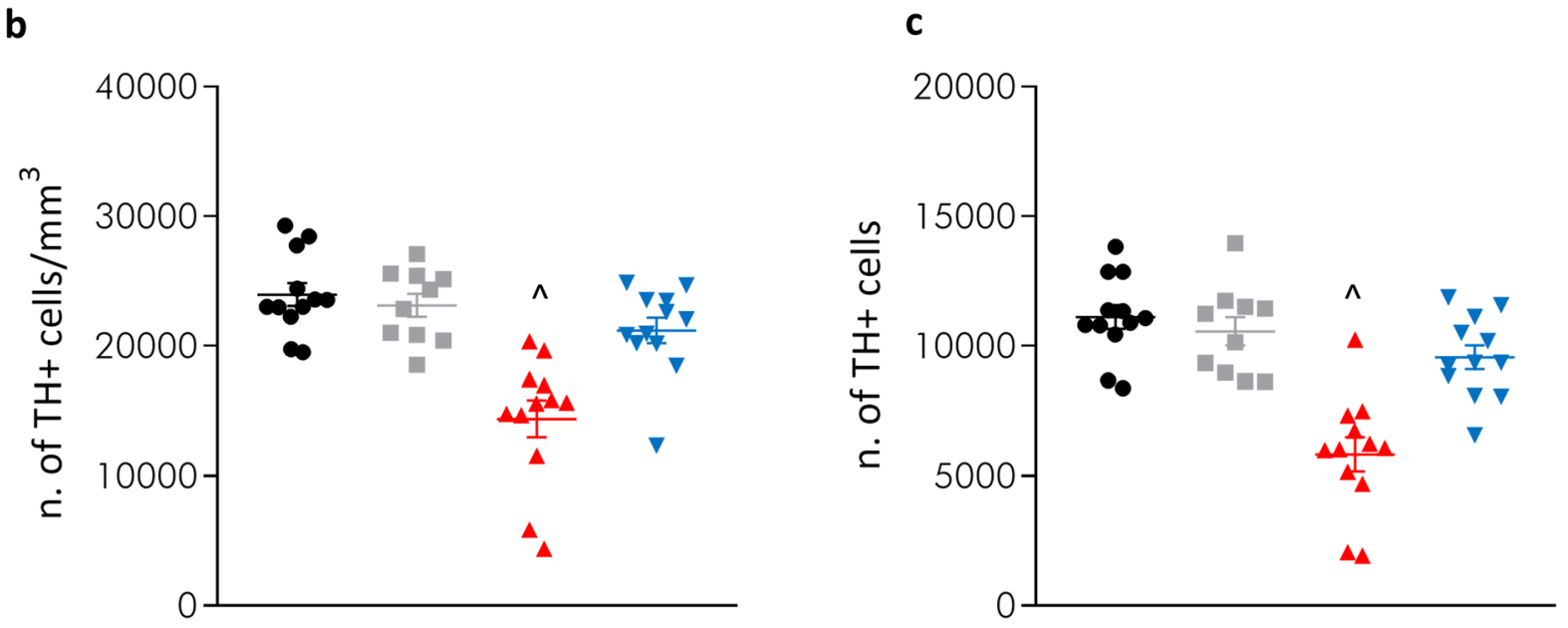

\section{NissI IR}
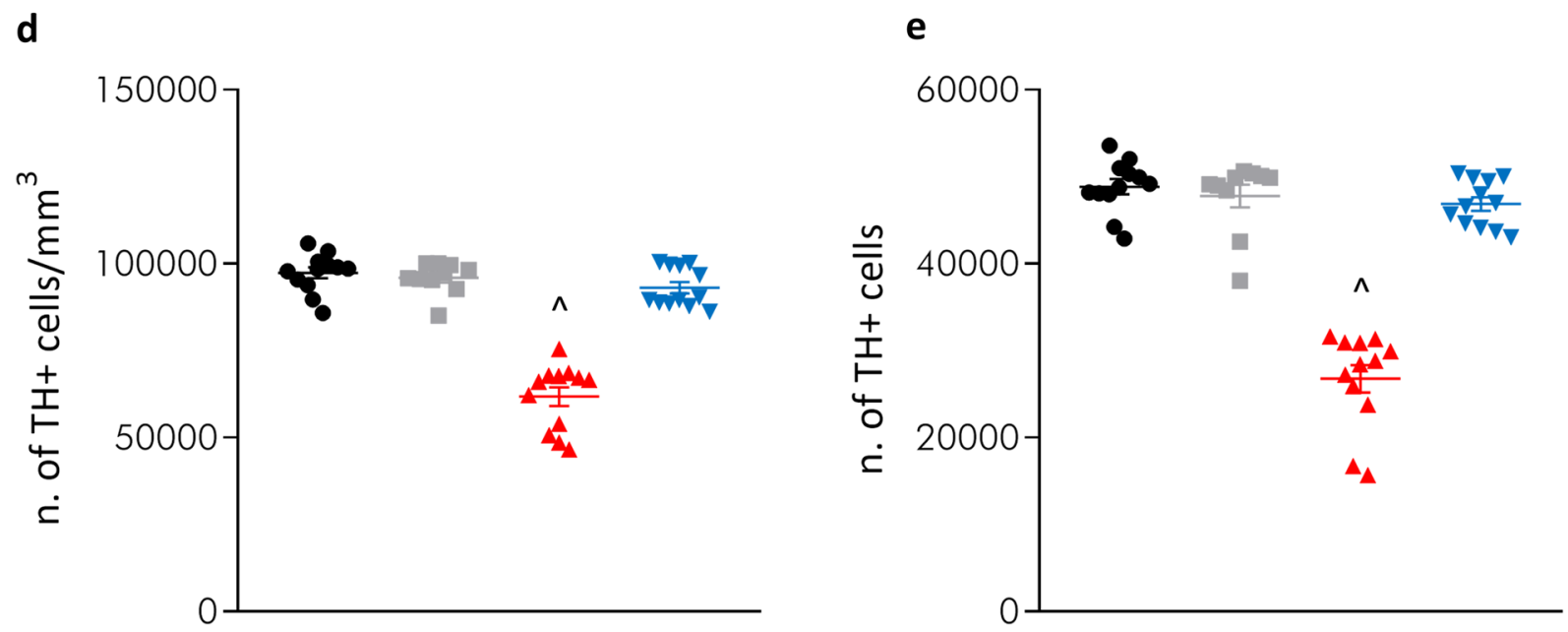
4Fig. $3 \mathrm{H}-\alpha$ SynOs induced a progressive nigrostriatal degeneration, which is rescued by pomalidomide treatment. a Representative images of TH-stained SNpc slices (magnification $5 \times$ ), scale bar: $500 \mu \mathrm{m}$. b Density (expressed as number of $\mathrm{TH}^{+}$cells $/ \mathrm{mm}^{3}$ ) and c number of $\mathrm{TH}^{+}$cells measured by stereological counting 3 months after the $\mathrm{H}-\alpha \mathrm{SynO}$ or vehicle infusion and after 2 months treatment with pomalidomide or Saline. (c-d) Stereological quantification of Nissl-stained cells in the SNpc. Values represent the mean \pm SEM. ${ }^{\wedge} p<0.001$ vs all other groups, by two-way ANOVA and Tukey's post hoc test

this aspect and to investigate how the $\alpha$ SynOs challenge and pomalidomide treatment affected the heterogeneity of cytokine content, data were expressed as a frequency distribution of cytokine volume within microglial cells (Fig. 5a, b). Whereas the histogram of the Veh-Sal group was characterized by a single peak/population that expressed low level of TNF- $\alpha$, the histogram of the H- $\alpha$ SynOs-Sal group showed two peaks, produced by the low TNF- $\alpha$ expressing cells and a new-high TNF- $\alpha$ expressing population. Remarkably, this second population was abolished in the $\mathrm{H}-\alpha \mathrm{SynOs}-\mathrm{Pom}$ group (Fig. 5a, $\mathrm{a}_{1}-\mathrm{a}_{3}$ ). These two populations of cells-low and high TNF- $\alpha$ labelled-that emerged from the deconvolution analysis, were separated by setting an appropriate cutoff value and statistically compared (Fig. 6a, b and corresponding images $a_{1}, a_{2}, b_{1}-b_{2}$ ). As shown in Fig. $6 a$, a significant difference was found among groups in the TNF- $\alpha$ content, both in the low and in the high labelled cells. Specifically, $\mathrm{H}-\alpha \mathrm{SynOs-Sal}$ rats showed a significant decrease of low labelled cells and an increase of highly labelled cells, as compared to control rats, suggesting that a subpopulation of microglia produced a supraphysiological amount of TNF- $\alpha$ (Fig. $6 \mathrm{a}, \mathrm{a}_{1}, \mathrm{a}_{2}$ ). In contrast, H- $\alpha$ SynOs-Pom-treated rats displayed similar labelling to the control group for both cell populations, thereby indicating that pomalidomide treatment fully counteracted the $\mathrm{H}-\alpha$ SynOs-induced increase above physiological values of the proinflammatory cytokine (Fig. 6a, $a_{1}, a_{2}$ ).

Microglia were further characterized by assessing the content of the anti-inflammatory cytokine IL-10 across experimental groups (Fig. 5b, $b_{1}-b_{3}$ ). As shown in Fig. 5b, histograms of all experimental groups were characterized by a large peak of cells showing low IL-10 labelling. However, the $\mathrm{H}-\alpha$ SynOs-Sal histogram displayed a slight left-shift, which indicates a decrease in cytokine content. Moreover, the $\mathrm{H}-\alpha$ SynOs-Pom histogram showed an additional small peak associated with higher IL-10 volumes. A statistical comparison of low and high labelled cells across experimental groups determined that the overall content of IL-10 was not affected by the $\mathrm{H}-\alpha$ SynOs infusion; however, pomalidomide significantly increased the IL-10 content in a subpopulation of microglia above control values (Fig. 6b, $b_{1}, b_{2}$ ).

The percentage comparison of pro- versus anti-inflammatory microglia within the entire population analyzed, demonstrated that the $\mathrm{H}-\alpha \mathrm{SynOs}$ infusion induced a dysregulated ratio towards the pro-inflammatory form, whereas pomalidomide corrected such imbalance by inhibiting TNF- $\alpha$ and boosting IL-10 production (Fig. 6d). Collectively, our results show that pomalidomide, while not abolishing microgliosis and microglia reactivity, affected the microglia phenotype in an allostatic manner, to restore an optimal pro-/anti-inflammatory balance.

\section{Pomalidomide Mitigates H-aSynOs-Induced Systemic Inflammation}

The intracerebral infusion of $\mathrm{H}-\alpha \mathrm{SynO}$ induced a systemic inflammation, as indicated by a profound dysregulation in serum levels of a panel of serum immune/inflammatory mediators, including cytokines and chemokines (Figs. 7 and 8). Specifically, serum levels of several pro-inflammatory molecules were elevated three months following the $\mathrm{H}-\alpha \mathrm{SynOs}$
Fig. 4 Microgliosis after $\mathrm{H}-\alpha$ SynOs infusion with and without pomalidomide treatment. a Stacked bar chart shows the total volume occupied by Iba- $1^{+}$cells in the SNpc. b Representative images of Iba- 1 cells (red, magnification 63X), scale bar: $20 \mu \mathrm{m}$. Values represent the mean \pm SEM. $\wedge^{\wedge} p<0.0001$ vs. Veh-Sal and Veh-Pom, by Kruskall-Wallis followed by Dunn's post hoc test

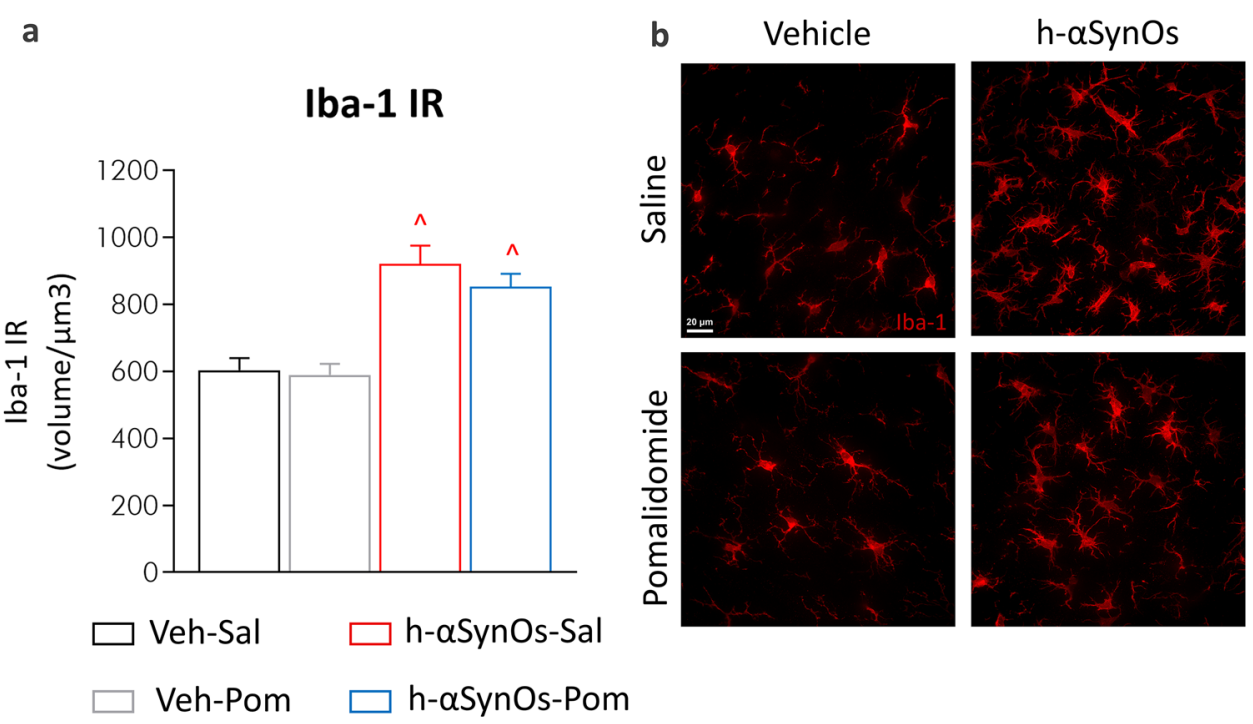




\section{TNF- $\alpha$}

a

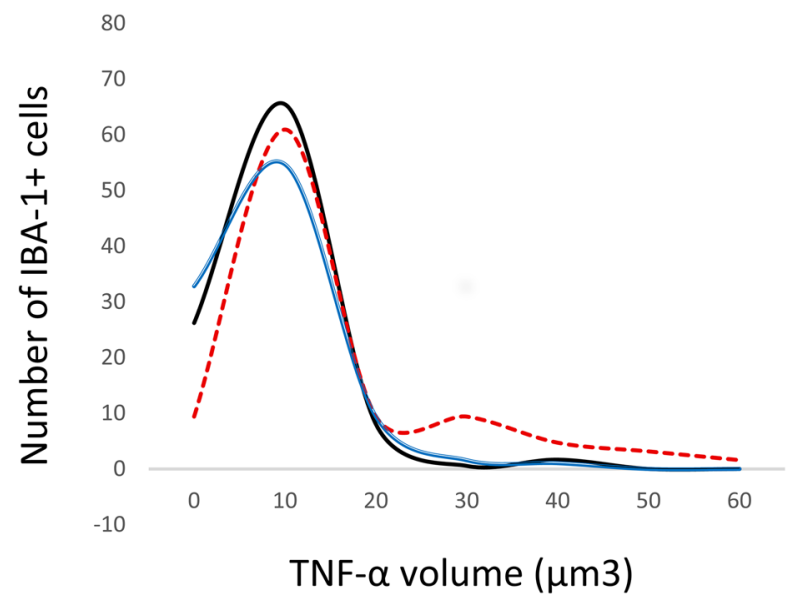

IL-10

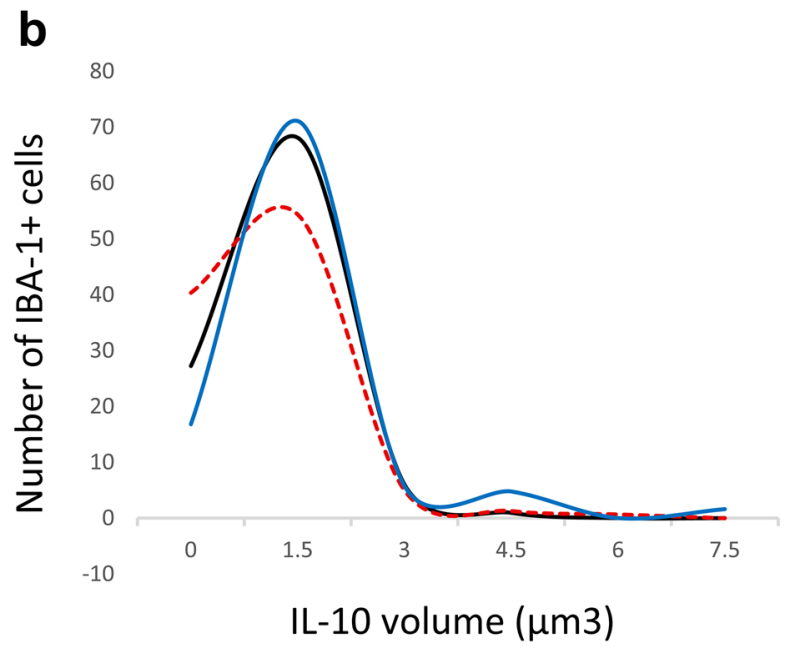

— Veh-Sal -.-- h- $\alpha$ SynOs-Sal — h- $\alpha$ SynOs-Pom
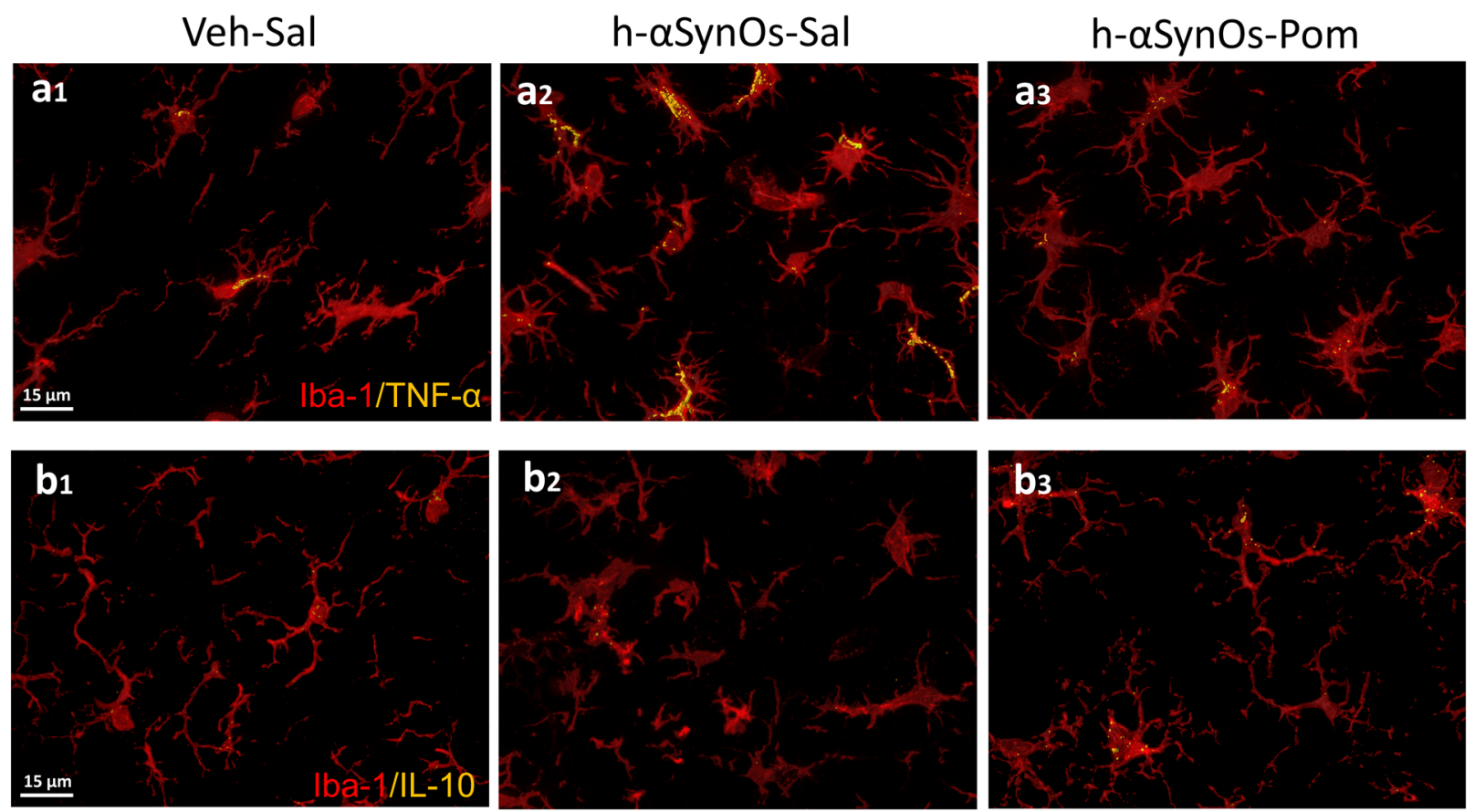

Fig. 5 Pomalidomide reverses the imbalance of cytokines expression in the SNpc of $\mathrm{H}-\alpha \mathrm{SynOs}$-infused rats. Frequency distribution of TNF- $\alpha$ (a) and IL-10 (b) colocalization within Iba-1 IR cells. Repre-

intracerebral infusion, including cytokines IL-1 $\beta$, IL-5, IL-6, IL-17, GCSF (Fig. 7), and chemokines RANTES, eotaxin, MCP3, CXCL 1/2, MCP1 (Fig. 8). Likewise notable, serum levels of the anti-inflammatory cytokine IL-10 as well as of IL-2 were decreased. Interestingly, serum TNF- $\alpha$ levels were not significantly affected by the infusion of $\alpha$ SynOs when sentative images of TNF- $\alpha$ (yellow) $\left(a_{1}-a_{3}\right)$ and IL-10 $\left(b_{1}-b_{3}\right)$ (yellow) colocalized with $\mathrm{Iba}^{-} 1^{+}$cells $(\mathrm{red})$. Magnification $63 \times$, scale bar: $15 \mu \mathrm{m}$

comparing the serum of vehicle-infused rats $(295.65 \mathrm{pg} / \mathrm{mL})$ vs. H- $\alpha$ SynOs-infused rats $(355.98 \mathrm{pg} / \mathrm{mL})$, suggesting a different regulation of this cytokine at the central and peripheral level. In line with these increases in several chemokines, we observed an increase in CD3 immunostaining in the SNpc of $\mathrm{H}-\alpha$ SynOs-infused rats, which did not colocalize with 


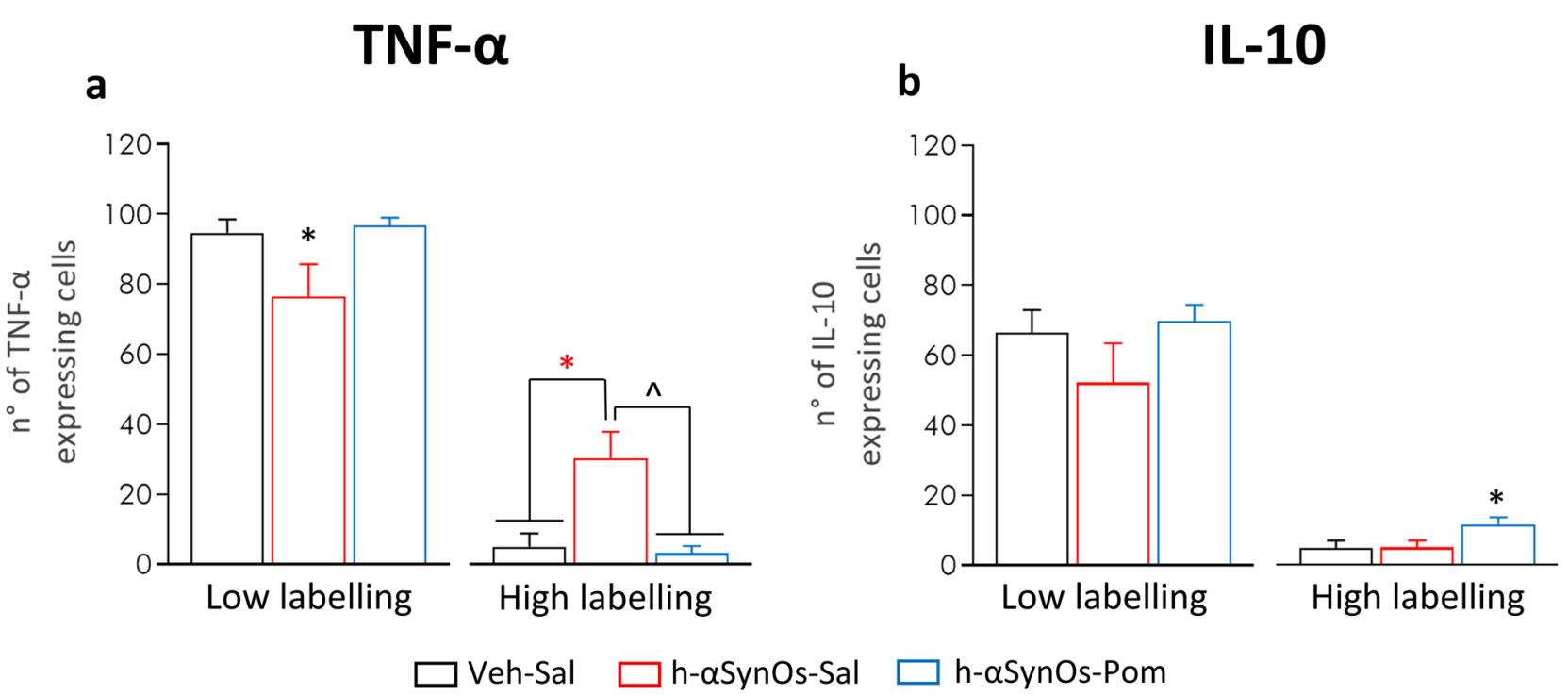

LOW LABELLING

TNF- $\alpha$
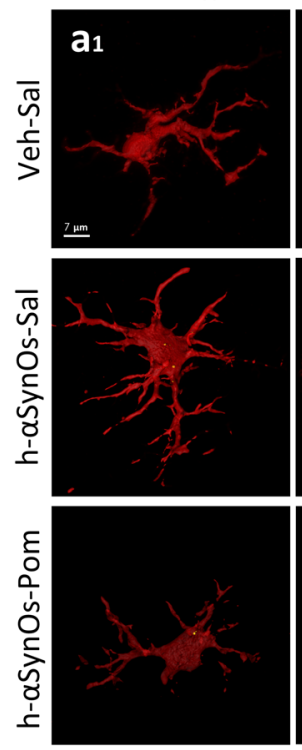

IL-10
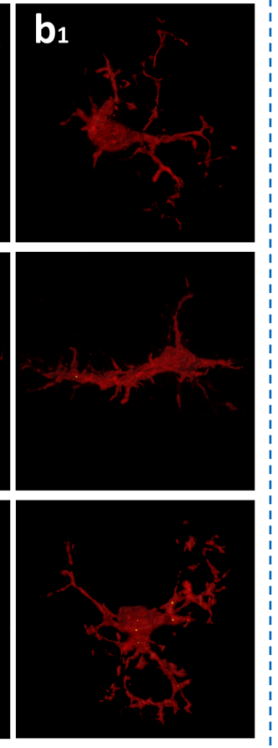

\section{HIGH LABELLING}

TNF- $\alpha$
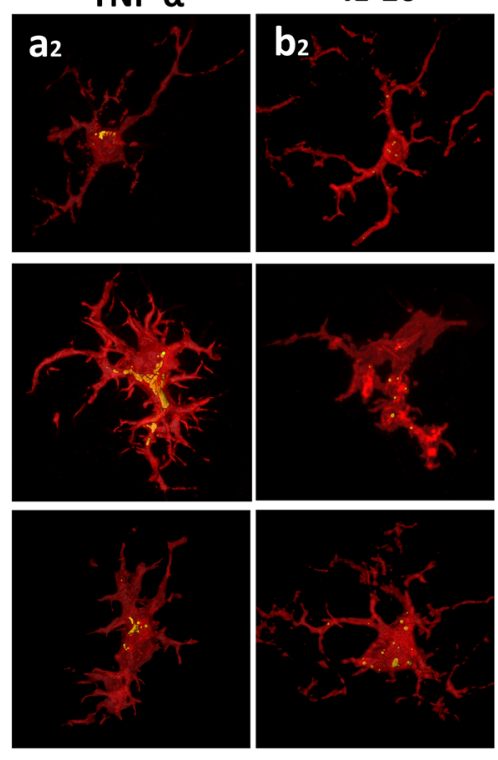

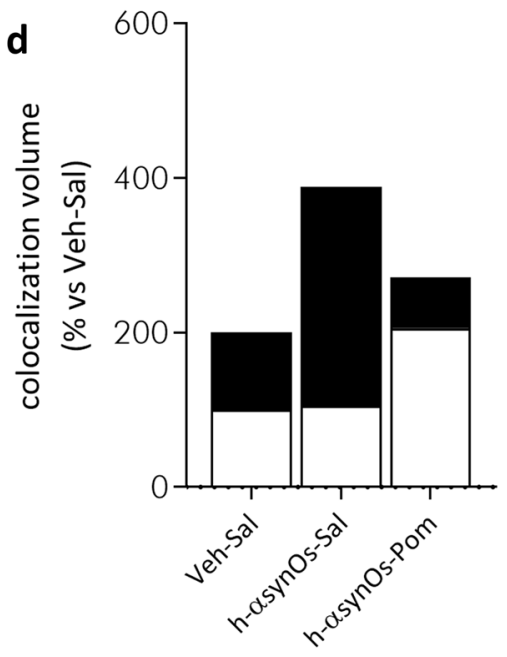

IL-10

TNF- $\alpha$
Fig. 6 The functional phenotype of Iba- $1^{+}$microglia is altered by $\mathrm{H}-\alpha$ SynOs infusion and pomalidomide treatment mitigates this. Iba$1^{+}$cells were categorized into two sub-populations, based on the (a) TNF- $\alpha$ and (b) IL-10 expression, namely low labelled and high labelled. Values represent the mean \pm SEM (one-way ANOVA fol-

Iba-1, suggesting peripheral T-cell infiltration into the brain parenchyma (Fig. 9). Notably, pomalidomide reversed most of the $\alpha$ SynOs-induced changes in the serum, by restoring the physiological levels of several inflammatory and lowed by Tukey's post hoc test). ${ }^{*} p<0.05 ; * p<0.01 ; \wedge p<0.001$. c Representative images of TNF- $\alpha$ (yellow) and IL-10 low (c) and high $\left(\mathrm{c}_{1}\right)$ expressing Iba- $1^{+}$cells (red). Magnification $63 \times$, scale bar: $7 \mu \mathrm{m}$. d Bar plot showing the relative percentage of each cytokine among the experimental groups

anti-inflammatory cytokines and chemokines, in line with the systemic immunomodulatory activity of this drug (Figs. 7 and 8). Accordingly, CD3 immunostaining was very low or absent in pomalidomide-treated rats (Fig. 9). 


\section{SERUM CYTOKINES}

IL-1 $\beta$

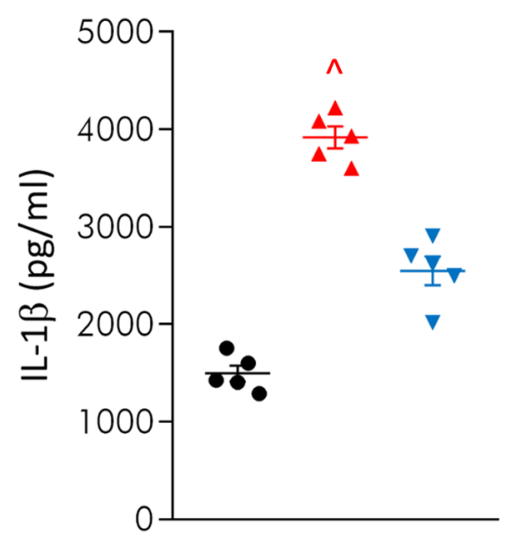

IL-10

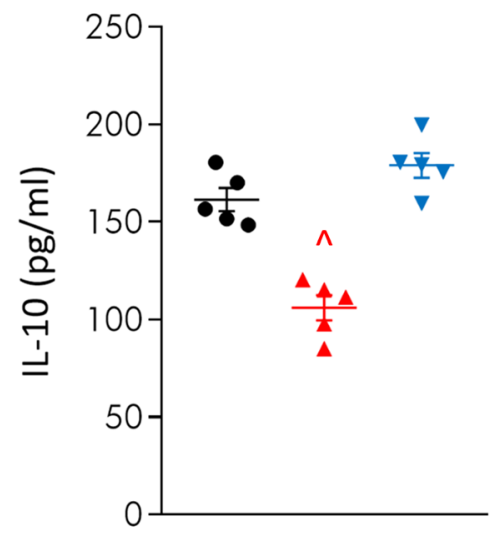

GCSF

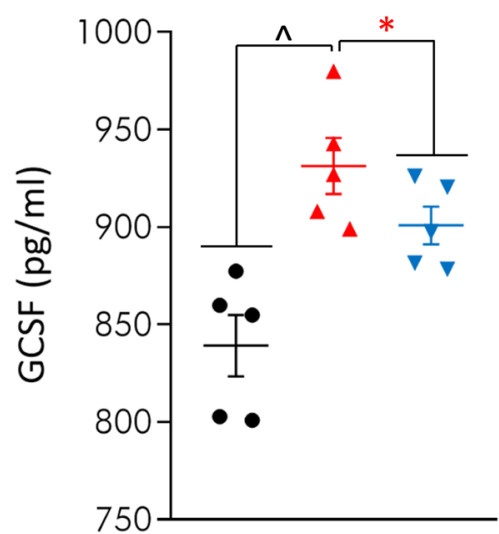

IL-5

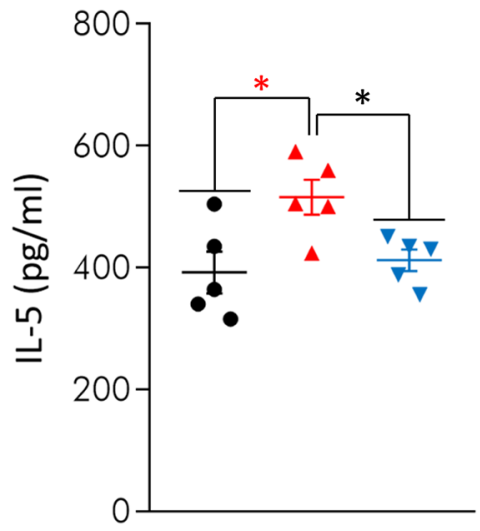

IL-2
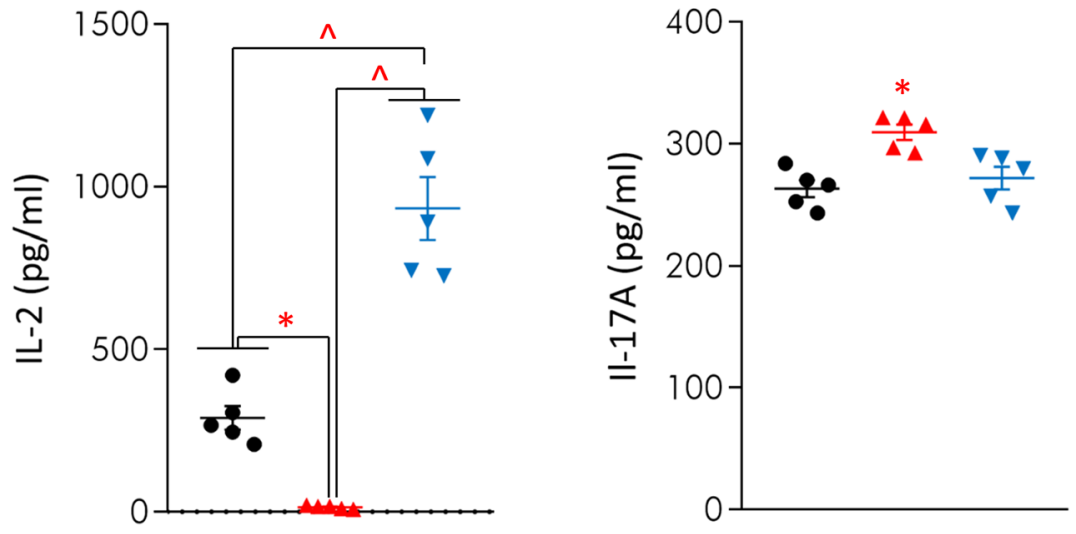

Fig. 7 Serum cytokine content after $\mathrm{H}-\alpha$ SynOs infusion and pomalidomide treatment. Serum cytokines were analyzed by multiplex ELISA. Values represent the mean \pm SEM. ${ }^{\wedge} p<0.0001 ;{ }^{\wedge} p<0.001 ;{ }^{*} p<0.01 ; * p<0.05$, by one-way ANOVA and Fisher's post hoc test 


\section{SERUM CHEMOKINES}

RANTES (CCL5)

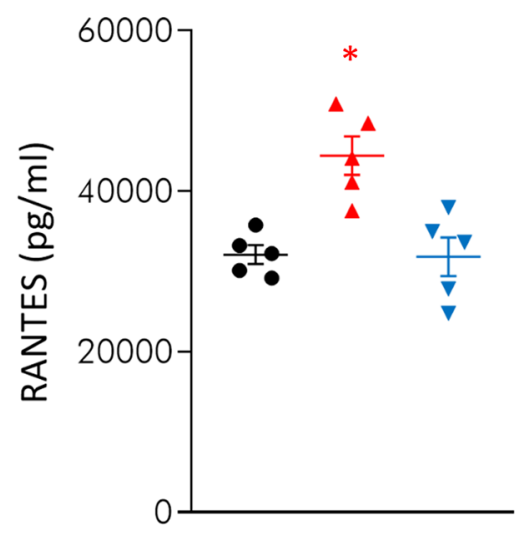

GRO $\alpha$ (CXCL1)

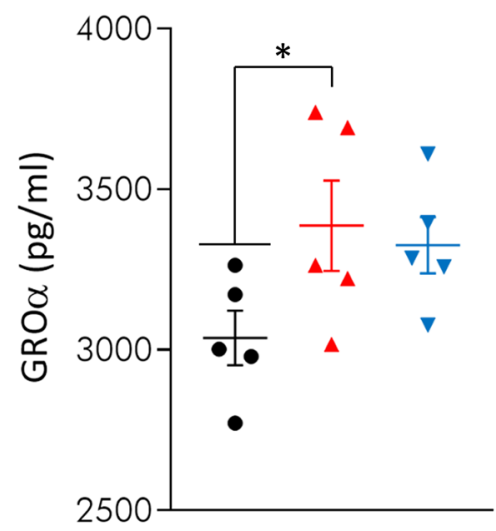

MCP1 (CCL2)

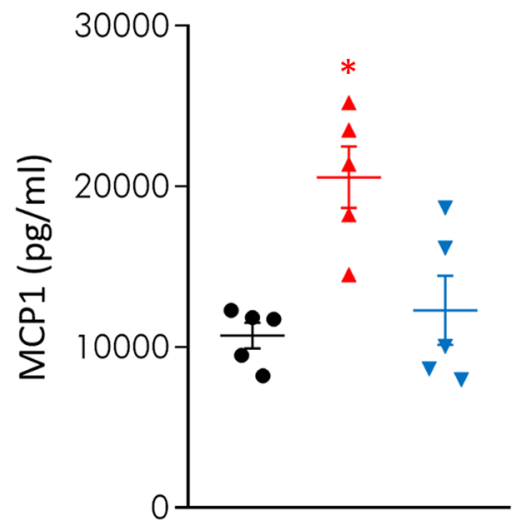

EOTAXIN

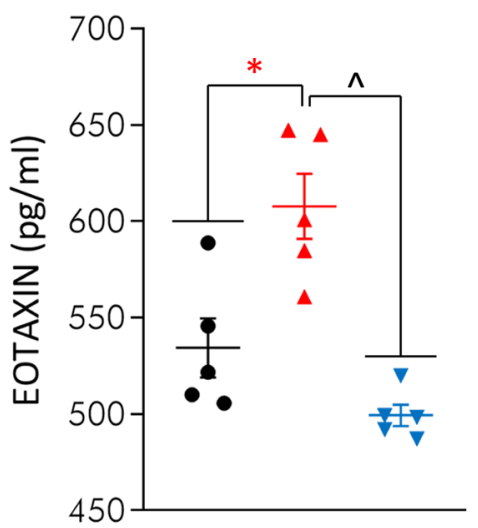

MIP2 $\alpha$ (CXCL2)

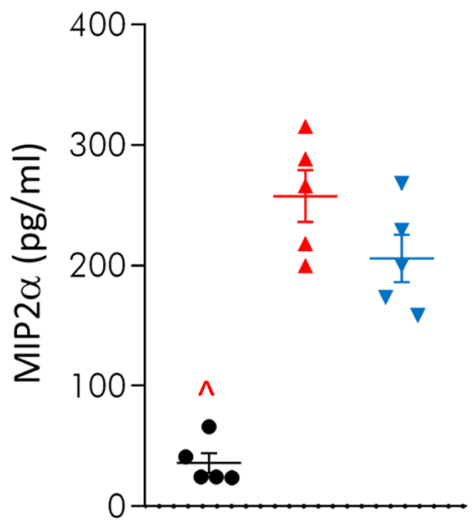

- Veh-Sal

$\Delta$ hOsyn-Sal

$\checkmark$ hOasyn-Pom

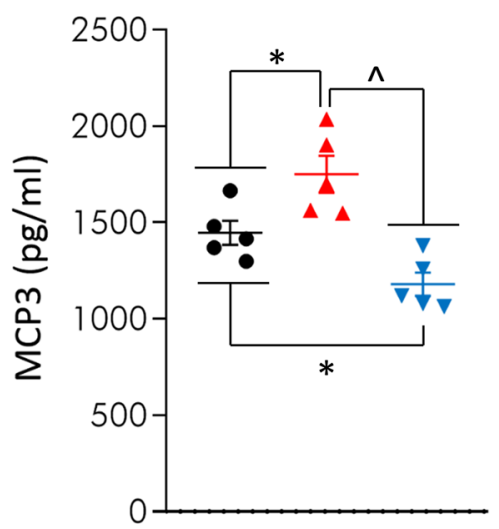

$\operatorname{MIP} 1 \alpha(\mathrm{CCL3})$

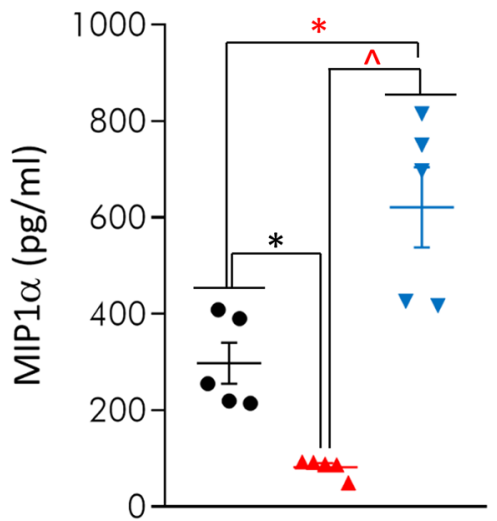

Fig. 8 Serum chemokine content after $\mathrm{H}-\alpha$ SynOs infusion and pomalidomide treatment. Serum chemokines were analyzed by multiplex ELISA. Values are the mean \pm SEM. ${ }^{\wedge} p<0.0001 ;{ }^{\wedge} p<0.001 ; * p<0.01 ; * p<0.05$, by one-way ANOVA followed by Fisher's post hoc test 
Fig. 9 Representative pictures of the SNpc showing $\mathrm{CD}^{+} \mathrm{T}$ cells infiltration after $\mathrm{H}-\alpha \mathrm{SynOs}$ infusion and after pomalidomide treatment $\left(\mathrm{a}-\mathrm{d}_{1}\right)$ Doubleimmunostaining was performed using anti-CD3 (green) and anti-Iba-1 (red) antibodies and DAPI staining (blue, nuclei). Magnification $63 \times$, scale bar: $20 \mu \mathrm{m}(\mathbf{a}-\mathbf{d})$ and $10 \mu \mathrm{m}\left(\mathrm{a}_{1}-\mathrm{d}_{1}\right)$
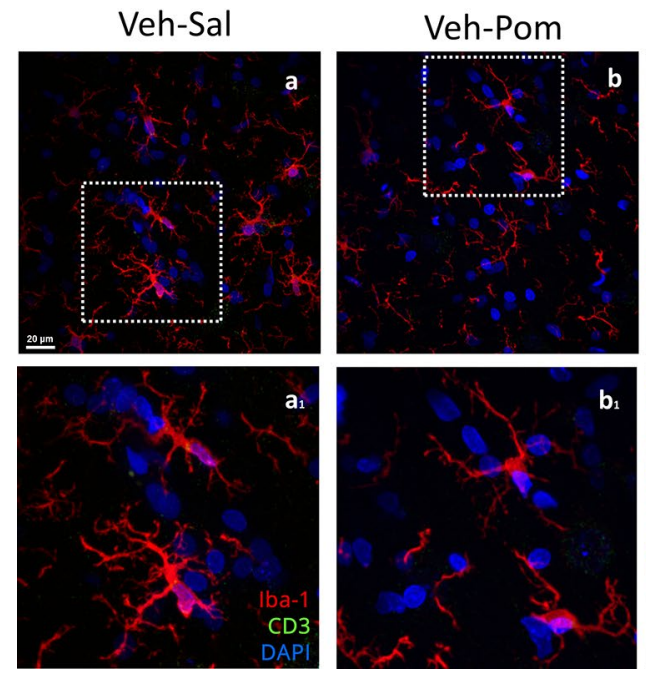

h- $\alpha$ SynOs-Sal
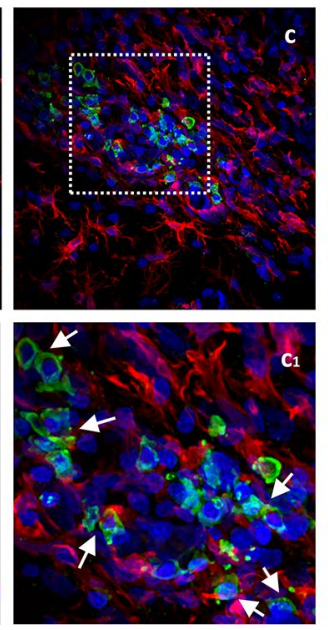

h- $\alpha$ SaynOs-Pom
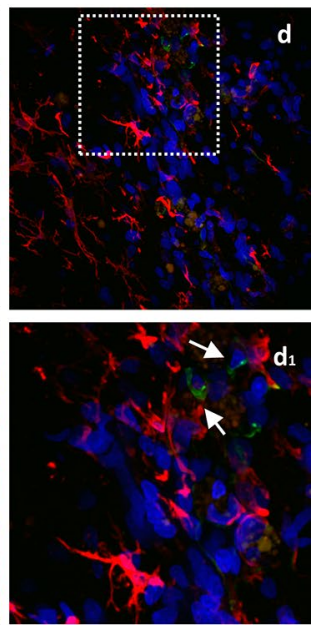

\section{Discussion}

In the present study, we took advantage of a recently validated mammalian model of PD based on the intranigral infusion of toxic $\mathrm{H}-\alpha \mathrm{SynOs}[29]$ to evaluate the diseasemodifying properties of the neuromodulatory IMiD drug pomalidomide. We found that pomalidomide was effective in mitigating the PD-related motor deficits and prevented the nigral dopaminergic cell loss induced by the intracerebral infusion of $\alpha$ SynOs. This effect was achieved by correcting the pathological phenotype of microglial cells to a more physiological-like one and, thereby, dampening the neurotoxic inflammatory response in the affected areas. Moreover, we demonstrated for the first time that the intracerebral infusion of $\alpha$ SynOs induced a systemic inflammatory response, characterized by a dysregulated cytokine/chemokine content in the serum, and associated with a peripheral cell infiltration within the SNC. Notably, pomalidomide reversed such a systemic pathological trait close to normality.

Immunomodulatory compounds are of increasing interest as a therapeutic strategy to target the neuroinflammatory component of $\mathrm{PD}$, with the aim of slowing the progression of the neurodegenerative processes [5]. Several FDA-approved neuromodulatory compounds, including fingolimod (Gilenya), tacrolimus (Fujimycin), cyclosporin, and rapamycin, have shown neuroprotective properties in rodent models of dopaminergic degeneration [44-48]. As a result, rapamycin has been prioritized as a therapeutic candidate to move into clinical trials for repurposing in $\mathrm{PD}$, based on solid preclinical evidence and its well-known neuromodulatory activity [49]. In contrast, IMiDs remain relatively poorly investigated in preclinical models of PD. Widely used and effective in cancer treatment, the relative dearth of preclinical research in PD models precludes their current proposition for clinical testing in this disorder. However, the latest generations of ImiDs offer several advantageous pharmacokinetic features. Notably, ImiDs are small molecular weight molecules that are orally bioavailable and have a greater brain uptake compared with classical immunosuppressants (brain/plasma concentration ratio rapamycin $<0.1$ ) [50] making them potentially more suitable for treating chronic neurological disorders [1].

Supporting repositioning of IMiDs in neurological disorders in general, several studies have reported the beneficial effects of thalidomide or thalidomide-derivatives in Alzheimer disease (AD) preclinical models [51-54]. These studies have led to the clinical assessment of thalidomide in a cohort of mild to moderate $\mathrm{AD}$ patients [55], and of the derivative lenalidomide in a clinical trial aimed at evaluating potential beneficial effects in patients with mild cognitive impairment (MCI) and AD [56]. As regards to PD, the protective potential of thalidomide was first reported in MPTP-intoxicated mice [57], and both thalidomide and lenalidomide were subsequently evaluated in $\alpha$-synuclein overexpressing mice, providing an improvement in both motor performance and neuropathological parameters [17]. Regrettably, despite preliminary promising results for the repurposing of IMiDs in PD, thalidomide and to a lesser extent lenalidomide, possess caveats that dampen enthusiasm for clinical translation in neurological disorders. Specifically, long-term use of both thalidomide and lenalidomide at doses required to mitigate inflammation has been associated with peripheral neuropathy and their wellknown teratogenic effects [25, 28, 58-60]. Indeed, the poor tolerability of thalidomide hampered escalation to the desired dose purported efficacious in a recent AD clinical trial, resulting in trial failure [55]. Moreover, lenalidomide, although more potent than thalidomide in inducing anti-inflammatory effects, is a reported substrate for the p-glycoprotein efflux pump at the BBB, which may limit its CNS penetrance and maintenance of CNS therapeutic levels [61].

Pomalidomide, a third-generation analogue of thalidomide, possesses favorable physicochemical characteristics to support 
its potential CNS use by being in line with the Lipinski rule of 5 , which predicts the successful delivery of a drug to the drug target after oral administration in physiological conditions [18]. In addition, pomalidomide possesses a promising CNS MPO (multiparameter optimization) score of 4.8 [1] that is predictive for an agent having desirable drug-like properties for neurological action. In previous studies, pomalidomide's administration intraperitoneally to rodents as a suspension resulted in a brain/plasma concentration ratio in the range of 0.39 to 0.71 [27, 62, 63], thereby positioning this drug in the first line for repurposing in neurological disorders. Here, the pomalidomide bioavailability was further improved by the administration of the drug as a nanosuspension, which increases both the brain/plasma ratio and the brain concentration of the drug (Cardia et al. submitted) and allows low doses more readily to provide an effective brain concentration. Moreover, pomalidomide is far more potent than thalidomide or lenalidomide toward TNF- $\alpha$ inhibitory action $[25,26]$, which allows a drug dosing less likely to generate teratogenic and neurotoxic adverse actions but with potent anti-inflammatory activity $[25,28]$. In the light of these features, pomalidomide is currently a first-choice drug for the treatment of select forms of tumors, with a dosing regimen 50-fold lower than thalidomide [64]. Up to the present study, pomalidomide has not been evaluated in mammalian models of PD. In a recent study, we reported its efficacy in a Drosophila LRRK2 $2^{\mathrm{WD} 40}$ genetic PD model $[16,65]$. In that study, we showed that LRRK2 ${ }^{\mathrm{WD} 40}$ flies develop motor impairment and gradual loss of dopaminergic neurons with age [16], and pomalidomide administration through the diet prevented both age-dependent motor impairment and neuronal loss [16]. Supporting the neuroprotective property of this drug, pomalidomide was effective in reducing the ischemic brain injury in both mice and rats [27]. Moreover, both pomalidomide and its new derivative 3,6'-dithiopomalidomide (3,6'-DP) reduced cell loss in primary dopaminergic neuron cultures exposed to $\alpha$ SynOs [19].

In the present study, the neuroprotective activity of pomalidomide was characterized for the first time in a mammalian neuropathological model of PD, taking advantage of a recently developed model obtained by the intranigral infusion of toxic $\mathrm{H}-\alpha \mathrm{SynOs}$ in the rat [29]. Here, $\alpha$ SynOs were infused bilaterally within the SNpc in order to best model the clinical motor symptoms of PD. $\alpha$-Syn-based models of PD reproduce critical pathological traits of the disease, epitomized by the $\alpha$-Syn-induced progressive degeneration of dopamine neurons, spreading of aggregated $\alpha$-Syn and a persistent inflammatory reaction in pathologically affected brain areas. These features position these models as highly applicable to study PD neuropathology and to test diseasemodifying molecules targeting pathological mechanisms relevant to the model [31]. Specifically, small $\alpha$-Syn aggregates that include oligomers, prefibrils and protofibrils are retained and, due to their degree of solubility, provide highly toxic species [66, 67]. Indeed, such soluble aggregates have been reported in degenerating areas within the brain and in biological fluids of PD patients [67-71]. In vitro and in vivo studies have suggested that oligomeric species induce the intracellular $\alpha$-Syn aggregation and, in turn, promote the spreading of protein aggregates $[31,72-76]$. Furthermore, $\alpha$ SynOs released from neurons establish contact with glial cells and stimulate pro-inflammatory responses in microglia $[29,77-81]$. The small $\mathrm{H}-\alpha \mathrm{SynO}$ os used in the present study offer an additional advantage of being highly homogeneous in size and structure, being subjected to a standardized protocol to generate highly pure oligomers with defined structural properties [30]. This feature confers a high degree of reproducibility both within and across experiments in terms of the extent of neurodegeneration, as previously shown [29], which is pivotal when evaluating and comparing neuroprotective strategies.

Assessing neuroprotection in the $\alpha$-Syn-based model used in the present study is, therefore, a crucial step to test molecules targeting the immune system in PD, as a prelude to clinical translation.

In line with our previous study [29], rats displayed significant motor deficits 3 months after the infusion surgery. Herein, we used three specific tests to probe different aspects of motor symptomatology, including sensorimotor deficits that were assessed via the beam challenging test and the gate test, and coordination impairment in fine movements execution that was evaluated via the vermicelli handling test $[38,39]$. These tests support reliable measurement of typical impairments similarly evident in human PD identified as gait disturbances, such as stepping falls and bradykinesia [82]. Specifically, the beam challenging test is a validated behavioral task highly sensitive for motor deficits associated with partial degeneration of the nigrostriatal pathway, which has been previously used to test rodents in PD models [35, 36, 83]. The infusion of $\alpha$ SynOs induced an increase in the number of errors committed in the beam challenge test, which was reversed by pomalidomide. In the gait test, oligomer-infused rats exhibited a decrease in cadence (steps/s) and an increase in the time spent to traverse a fixed distance, expressed as a decreased velocity. Moreover, oligomer-infused rats displayed irregular, shorter strides that reflect the shortening in step amplitude in PD. Notably, all these motor impairments were mitigated by pomalidomide. Finally, the vermicelli handling test revealed an impairment in fine movements, which was again reversed by pomalidomide. In entirety, the results strongly forecast a beneficial action for pomalidomide in PD motor symptomatology.

Importantly, the efficacy of pomalidomide was underpinned by the rescue of dopaminergic cell loss, as revealed by the stereological count of nigral neurons. This analysis 
confirmed a significant cell loss in the $\alpha$ Syn-infused SNpc and the neuroprotective action of pomalidomide. The associated stereological count of Nissl-stained cells is of relevance as it confirms that changes in TH staining reflected changes in neuron number. It should be noted that pomalidomide treatment was initiated 1 month after $\mathrm{H}-\alpha \mathrm{SynOs}$ infusion, a time-point that may be considered to model the prodromal phase of $\mathrm{PD}$, when mitochondrial damage and neuroinflammation are not yet associated with neuronal loss [29]. Therefore, the results of the present study suggest that pomalidomide can mitigate already initiated and ongoing neurodegenerative processes. This is pivotal in view of the translational relevance of this study, which suggests a potential disease-modifying effect of the drug treatment if given to early-diagnosed PD patients.

IMiDs are immunomodulatory compounds with potent anti-inflammatory properties, which were manifested in the present study by a dampening of the inflammatory response both within the brain and in peripheral blood. Intracerebral $\alpha$ SynOs infusion induced a chronic microglial reaction, revealed by an increase in Iba-1 IR, as previously shown in this as well as in other's PD models, and in accord with the persistent microgliosis reported in the brain of PD patients [29, 83-88]. Moreover, reactive microglia displayed a pathological gain of toxic functions suggested by the increased production of the proinflammatory cytokine TNF- $\alpha$, in line with an altered cytokine production, and elevations in reported levels in the cerebrospinal fluid of PD patients [89-91]. Notably, whereas in control rats the majority of microglia displayed low physiological levels of TNF- $\alpha$, in oligomer-infused rats, two microglia subpopulations were recognized, displaying low and high TNF- $\alpha$ content, respectively, suggesting a supraphysiological production of the cytokine. Importantly, pomalidomide treatment suppressed the high TNF- $\alpha$ expressing microglia population leaving unaltered the low TNF- $\alpha$ expressing population, thereby restoring physiological levels of the cytokine. Moreover, pomalidomide augmented the microglia production of the anti-inflammatory cytokine IL-10, as expected by the immunomodulatory action of this drug. In synopsis, pomalidomide provided an allostatic regulation of microglia phenotype to restore the physiological pro/anti-inflammatory balance.

Of interest and, to our knowledge, not previously described in studies of intracerebral $\alpha$-synuclein oligomer infusion was the development of a long-term peripheral inflammatory condition, characterized by the increased serum content of inflammatory cytokines and the concomitant decrease of anti-inflammatory mediators $[92,93]$. This result is in line with an increasing literature reporting impaired serum cytokines in PD patients, and with the concept of PD as a systemic rather than CNS-specific disease, further validating our model as a translational model of PD
[15]. Although the origin of systemic inflammation in PD remains uncertain, our results suggest that it may originate from the presence of toxic $\alpha$-synuclein oligomers within the $\mathrm{CNS}$, leaving an open question as to the sequence of mechanistic events.

Although there is a convergence of opinion on serum cytokine dysregulation in PD, conflicting findings have been reported with respect to single cytokines [94, 95]. Nonetheless, the elevated serum level of IL-17 present in our intracerebrally $\alpha$-synuclein-infused rats is worthy of note, and is in line with the pivotal role currently attributed to this cytokine in neurodegenerative diseases and in glial cell activation [96]. Moreover, in line with a prevalence of the inflammatory immunophenotype in PD, IL-6 was significantly elevated, whereas IL-10 was significantly decreased in our model $[15,92,93]$. Finally, chemokines CXCL1/2 and RANTES were increased, in line with infiltration of immune cells into the inflammatory site, as revealed by CD3 immunostaining in $\alpha$ SynOs-infused rats [14, 97-99]. Interestingly, serum TNF- $\alpha$ was unchanged following intracerebral administration of $\alpha$ SynOs. Similarly, in a previous study, we found unchanged levels of serum TNF- $\alpha$ after dopamine depletion and after a chronic L-DOPA treatment, despite an intense inflammatory response within the brain [41]. Importantly, pomalidomide dampened the systemic inflammation, largely restoring normal cytokine levels within the serum. Pomalidomide was therefore able to effectively target the inflammation present in this PD model, resetting both the central and peripheral components to physiological, rather than pathological levels, and mitigating dopaminergic neuron losses and motor impairments.

Altogether, the present study provides compelling evidence of pomalidomide's disease-modifying potential in a translational rodent model of PD to aid bridge the gap between preclinical and clinical studies, and fosters the repurposing of this drug for clinical testing in PD patients.

Supplementary Information The online version contains supplementary material available at https://doi.org/10.1007/s13311-022-01182-2.

Acknowledgements This research was supported in part by (i) the Intramural Research Program at the University of Cagliari, Italy; (ii) the Intramural Research Program of the National Institute on Aging, National Institutes of Health, Baltimore, MD, USA; (iii) European Research Council, Award number: ERC CoG BioDisOrder 819644.

Required Author Forms Disclosure forms provided by the authors are available with the online version of this article.

Open Access This article is licensed under a Creative Commons Attribution 4.0 International License, which permits use, sharing, adaptation, distribution and reproduction in any medium or format, as long as you give appropriate credit to the original author(s) and the source, provide a link to the Creative Commons licence, and indicate if changes 
were made. The images or other third party material in this article are included in the article's Creative Commons licence, unless indicated otherwise in a credit line to the material. If material is not included in the article's Creative Commons licence and your intended use is not permitted by statutory regulation or exceeds the permitted use, you will need to obtain permission directly from the copyright holder. To view a copy of this licence, visit http://creativecommons.org/licenses/by/4.0/.

\section{References}

1. Jung YJ, Tweedie D, Scerba MT, Kim DS, Palmas MF, Pisanu A, et al. Repurposing immunomodulatory Imide Drugs (IMiDs) in neuropsychiatric and neurodegenerative disorders. Front Neurosci. 2021;15:656921.

2. McFarthing K, Rafaloff G, Baptista MAS, Wyse RK, Stott SRW. Parkinson's disease drug therapies in the clinical trial pipeline: 2021 update. J Parkinsons Dis. 2021;11:891-903.

3. Pushpakom S, Iorio F, Eyers PA, Escott KJ, Hopper S, Wells A, et al. Drug repurposing: progress, challenges and recommendations. Nat Rev Drug Discov. 2019;18:41-58.

4. Stott SRW, Wyse RK, Brundin P. Drug repurposing for Parkinson's disease: the international linked clinical trials experience. Front Neurosci. 2021;15:653377.

5. Martinez B, Peplow PV. Neuroprotection by immunomodulatory agents in animal models of Parkinson's disease. Neural Regen Res. 2018;13:1493-506.

6. Liu C-Y, Wang X, Liu C, Zhang H-L. Pharmacological targeting of microglial activation: new therapeutic approach. Front Cell Neurosci. 2019;13:514.

7. Villar-Piqué A, Lopes da Fonseca T, Outeiro TF. Structure, function and toxicity of alpha-synuclein: the Bermuda triangle in synucleinopathies. J Neurochem. 2016;139 Suppl:240-55.

8. Kuter KZ, Cenci MA, Carta AR. The role of glia in Parkinson's disease: emerging concepts and therapeutic applications. Prog Brain Res. 2020;252:131-68.

9. Parpura V, Heneka MT, Montana V, Oliet SHR, Schousboe A, Haydon PG, et al. Glial cells in (patho)physiology. J Neurochem. 2012;121:4-27.

10. Pekny M, Pekna M, Messing A, Steinhäuser C, Lee J-M, Parpura V, et al. Astrocytes: a central element in neurological diseases. Acta Neuropathol. 2016;131:323-45.

11. Troncoso-Escudero P, Parra A, Nassif M, Vidal RL. Outside in: unraveling the role of neuroinflammation in the progression of Parkinson's disease. Front Neurol. 2018;9:860.

12. Verkhratsky A, Zorec R. Astroglial signalling in health and disease. Neurosci Lett. 2019;689:1-4.

13. Brodacki B, Staszewski J, Toczyłowska B, Kozłowska E, Drela N, Chalimoniuk M, et al. Serum interleukin (IL-2, IL-10, IL-6, IL-4), TNFalpha, and INFgamma concentrations are elevated in patients with atypical and idiopathic parkinsonism. Neurosci Lett. 2008;441:158-62.

14. Qin X-Y, Zhang S-P, Cao C, Loh YP, Cheng Y. Aberrations in peripheral inflammatory cytokine levels in Parkinson disease: a systematic review and meta-analysis. JAMA Neurol. 2016;73:1316-24.

15. Pajares M, I Rojo A, Manda G, Boscá L, Cuadrado A. Inflammation in Parkinson's disease: mechanisms and therapeutic implications. Cells. 2020;9.

16. Casu MA, Mocci I, Isola R, Pisanu A, Boi L, Mulas G, et al. Neuroprotection by the immunomodulatory drug pomalidomide in the Drosophila LRRK2WD40 genetic model of Parkinson's disease. Front Aging Neurosci. 2020;12.

17. Valera E, Mante M, Anderson S, Rockenstein E, Masliah E. Lenalidomide reduces microglial activation and behavioral deficits in a transgenic model of Parkinson's disease. J Neuroinflammation. 2015;12.
18. Banks WA, Greig NH. Small molecules as central nervous system therapeutics: old challenges, new directions, and a philosophic divide. Future Med Chem. 2019;11:489-93.

19. Lin CT, Lecca D, Yang LY, Luo W, Scerba MT, Tweedie D, et al. 3,6'-dithiopomalidomide reduces neural loss, inflammation, behavioral deficits in brain injury and microglial activation. Elife. 2020;9:1-78.

20. Chanan-Khan AA, Swaika A, Paulus A, Kumar SK, Mikhael JR, Rajkumar S V, et al. Pomalidomide: the new immunomodulatory agent for the treatment of multiple myeloma. Blood Cancer J. 2013;3:e143.

21. Moreira AL. Thalidomide exerts its inhibitory action on tumor necrosis factor alpha by enhancing mRNA degradation. 1993;177.

22. Sampaio EP, Sarno EN, Galilly R, Cohn ZA, Kaplan G. Thalidomide selectively inhibits tumor necrosis factor alpha production by stimulated human monocytes. J Exp Med. 1991;173:699-703.

23. Terpos E, Kanellias N, Christoulas D, Kastritis E, Dimopoulos MA. Pomalidomide: a novel drug to treat relapsed and refractory multiple myeloma. Onco Targets Ther. 2013;6:531-8.

24. Tweedie D, Frankola KA, Luo W, Li Y, Greig NH. Thalidomide analogues suppress lipopolysaccharide-induced synthesis of TNF- $\alpha$ and nitrite, an intermediate of nitric oxide, in a cellular model of Inflammation. Open Biochem J. 2011;5:37-44.

25. Mahony C, Erskine L, Niven J, Greig NH, Figg WD, Vargesson N. Pomalidomide is nonteratogenic in chicken and zebrafish embryos and nonneurotoxic in vitro. Proc Natl Acad Sci USA. 2013;110:12703-8.

26. Wang JY, Huang YN, Chiu CC, Tweedie D, Luo W, Pick CG, et al. Pomalidomide mitigates neuronal loss, neuroinflammation, and behavioral impairments induced by traumatic brain injury in rat. J Neuroinflammation. 2016;13.

27. Tsai Y-R, Tweedie D, Navas-Enamorado I, Scerba MT, Chang C-F, Lai J-H, et al. Pomalidomide reduces ischemic brain injury in rodents. Cell Transplant. 2019;28:096368971985007.

28. Vargesson N, Mahony C, Erskine L, Niven J, Greig NH, Figg WD. Reply to D'Amato et al. and Zeldis et al.: Screening of thalidomide derivatives in chicken and zebrafish embryos. Proc Natl Acad Sci USA. 2013;110:E4820.

29. Boi L, Pisanu A, Palmas MF, Fusco G, Carboni E, Casu MA, et al. Modeling Parkinson's disease neuropathology and symptoms by intranigral inoculation of preformed human $\alpha$-synuclein oligomers. Int J Mol Sci. 2020;21.

30. Fusco G, Chen SW, Williamson PTF, Cascella R, Perni M, Jarvis JA, et al. Structural basis of membrane disruption and cellular toxicity by $\alpha$-synuclein oligomers. Science. 2017;358:1440-3.

31. Carta AR, Boi L, Pisanu A, Palmas MF, Carboni E, De Simone A. Advances in modelling alpha-synuclein-induced Parkinson's diseases in rodents: virus-based models versus inoculation of exogenous preformed toxic species. J Neurosci Methods. 2020;338:108685.

32. Savitt D, Jankovic J. Targeting $\alpha$-synuclein in Parkinson's disease: progress towards the development of disease-modifying therapeutics. Drugs. 2019;79:797-810.

33. Paxinos $\mathrm{G}$, Watson $\mathrm{C}$. The rat brain in stereotaxic coordinates: hard cover edition. Elsevier. 2006.

34. Lai F, Schlich M, Pireddu R, Corrias F, Fadda AM, Sinico C. Production of nanosuspensions as a tool to improve drug bioavailability: focus on topical delivery. Curr Pharm Des. 2015;21:6089-103.

35. Drucker-Colín R, García-Hernández F. A new motor test sensitive to aging and dopaminergic function. J Neurosci Methods. 1991;39:153-61.

36. Fleming SM, Salcedo J, Fernagut P-O, Rockenstein E, Masliah E, Levine MS, et al. Early and progressive sensorimotor anomalies in mice overexpressing wild-type human alpha-synuclein. J Neurosci. 2004;24:9434-40.

37. Korecka JA, Moloney EB, Eggers R, Hobo B, Scheffer S, RasVerloop N, et al. Repulsive guidance molecule a (RGMa) induces 
neuropathological and behavioral changes that closely resemble Parkinson's disease. J Neurosci. 2017;37:9361-79.

38. Allred RP, Adkins DL, Woodlee MT, Husbands LC, Maldonado MA, Kane JR, et al. The vermicelli handling test: a simple quantitative measure of dexterous forepaw function in rats. J Neurosci Methods. 2008;170:229-44.

39. Tennant KA, Asay AL, Allred RP, Ozburn AR, Kleim JA, Jones TA. The vermicelli and capellini handling tests: simple quantitative measures of dexterous forepaw function in rats and mice. J Vis Exp. 2010.

40. Reynolds S, Millette A, Devine DP. Sensory and motor characterization in the postnatal valproate rat model of autism. Dev Neurosci. 2012;34:258-67.

41. Mulas G, Espa E, Fenu S, Spiga S, Cossu G, Pillai E, et al. Differential induction of dyskinesia and neuroinflammation by pulsatile versus continuous 1-DOPA delivery in the 6-OHDA model of Parkinson's disease. Exp Neurol. 2016;286:83-92.

42. Lecca D, Janda E, Mulas G, Diana A, Martino C, Angius F, et al. Boosting phagocytosis and anti-inflammatory phenotype in microglia mediates neuroprotection by PPAR $\gamma$ agonist MDG548 in Parkinson's disease models. Br J Pharmacol. 2018;175:3298-314.

43. Mouton PR, Gokhale AM, Ward NL, West MJ. Stereological length estimation using spherical probes. J Microsc. 2002;206:54-64.

44. Ren M, Han M, Wei X, Guo Y, Shi H, Zhang X, et al. FTY720 Attenuates 6-OHDA-associated dopaminergic degeneration in cellular and mouse parkinsonian models. Neurochem Res. 2017;42:686-96.

45. Tamburrino A, Churchill MJ, Wan OW, Colino-Sanguino Y, Ippolito R, Bergstrand $\mathrm{S}$, et al. Cyclosporin promotes neurorestoration and cell replacement therapy in pre-clinical models of Parkinson's disease. Acta Neuropathol Commun. 2015;3:84.

46. Van der Perren A, Macchi F, Toelen J, Carlon MS, Maris M, de Loor H, et al. FK506 reduces neuroinflammation and dopaminergic neurodegeneration in an $\alpha$-synuclein-based rat model for Parkinson's disease. Neurobiol Aging. 2015;36:1559-68.

47. Vidal-Martínez G, Vargas-Medrano J, Gil-Tommee C, Medina D, Garza NT, Yang B, et al. FTY720/fingolimod reduces synucleinopathy and improves gut motility in A53T mice: contributions of pro-brain-derived neurotrophic factor (pro-BDNF) and mature BDNF. J Biol Chem. 2016;291:20811-21.

48. Zhao P, Yang X, Yang L, Li M, Wood K, Liu Q, et al. Neuroprotective effects of fingolimod in mouse models of Parkinson's disease. FASEB J Off Publ Fed Am Soc Exp Biol. 2017;31:172-9.

49. Brundin P, Barker RA, Conn PJ, Dawson TM, Kieburtz K, Lees AJ, et al. Linked clinical trials-the development of new clinical learning studies in Parkinson's disease using screening of multiple prospective new treatments. J Parkinsons Dis. 2013;3:231-9.

50. Brandt C, Hillmann P, Noack A, Römermann K, Öhler LA, Rageot $\mathrm{D}$, et al. The novel, catalytic mTORC1/2 inhibitor PQR620 and the $\mathrm{PI} 3 \mathrm{~K} / \mathrm{mTORC} 1 / 2$ inhibitor PQR530 effectively cross the bloodbrain barrier and increase seizure threshold in a mouse model of chronic epilepsy. Neuropharmacology. 2018;140:107-20.

51. Gabbita SP, Srivastava MK, Eslami P, Johnson MF, Kobritz NK, Tweedie D, et al. Early intervention with a small molecule inhibitor for tumor necrosis factor- $\alpha$ prevents cognitive deficits in a triple transgenic mouse model of Alzheimer's disease. J Neuroinflammation. 2012;9:1-16.

52. Ryu JK, McLarnon JG. Thalidomide inhibition of perturbed vasculature and glial-derived tumor necrosis factor- $\alpha$ in an animal model of inflamed Alzheimer's disease brain. Neurobiol Dis. 2008;29:254-66.

53. Tweedie D, Ferguson RA, Fishman K, Frankola KA, Van Praag $\mathrm{H}$, Holloway HW, et al. Tumor necrosis factor- $\alpha$ synthesis inhibitor 3,6'-dithiothalidomide attenuates markers of inflammation, Alzheimer pathology and behavioral deficits in animal models of neuroinflammation and Alzheimer's disease. J Neuroinflammation. 2012;9:106.
54. Zhu X, Giordano T, Yu QS, Holloway HW, Perry TA, Lahiri $\mathrm{DK}$, et al. Thiothalidomides: novel isosteric analogues of thalidomide with enhanced TNF- $\alpha$ inhibitory activity. J Med Chem. 2003;46:5222-9.

55. Decourt B, Drumm-Gurnee D, Wilson J, Jacobson S, Belden C, Sirrel S, et al. Poor safety and tolerability hamper reaching a potentially therapeutic dose in the use of thalidomide for Alzheimer's disease: results from a double-blind, placebo-controlled trial. Curr Alzheimer Res. 2017;14:1-1.

56. Decourt B, Wilson J, Ritter A, Dardis C, DiFilippo FP, Zhuang $\mathrm{X}$, et al. MCLENA-1: a phase II clinical trial for the assessment of safety, tolerability, and efficacy of lenalidomide in patients with mild cognitive impairment due to Alzheimer's disease. Open Access J Clin Trials. 2020;12:1-13.

57. Ferger B, Leng A, Mura A, Hengerer B, Feldon J. Genetic ablation of tumor necrosis factor-alpha (TNF- $\alpha)$ and pharmacological inhibition of TNF-synthesis attenuates MPTP toxicity in mouse striatum. J Neurochem. 2004;89:822-33.

58. Christian MS, Laskin OL, Sharper V, Hoberman A, Stirling DI, Latriano L. Evaluation of the developmental toxicity of lenalidomide in rabbits. Birth Defects Res B Dev Reprod Toxicol. 2007;80:188-207.

59. Voorhees PM, Laubach J, Anderson KC, Richardson PG. Peripheral neuropathy in multiple myeloma patients receiving lenalidomide, bortezomib, and dexamethasone (RVD) therapy. Blood. 2013;121:858.

60. Palumbo A, Waage A, Hulin C, Beksac M, Zweegman S, Gay F, et al. Safety of thalidomide in newly diagnosed elderly myeloma patients: a meta-analysis of data from individual patients in six randomized trials. Haematologica. 2013;98:87-94.

61. Hughes JH, Upton RN, Reuter SE, Rozewski DM, Phelps MA, Foster DJR. Development of a physiologically based pharmacokinetic model for intravenous lenalidomide in mice. Cancer Chemother Pharmacol. 2019;84:1073-87.

62. Jiang Y, Wang J, Rozewski DM, Kolli S, Wu C-H, Chen C-S, et al. Sensitive liquid chromatography/mass spectrometry methods for quantification of pomalidomide in mouse plasma and brain tissue. J Pharm Biomed Anal. 2014;88:262-8.

63. European Medicines Agency. Committee for medicinal products for human use, assessment report: pomalidomide celgene. Procedure No. EMEA/H/C/002682, 2013. n.d.

64. Mo CC, Richardson PG. Pomalidomide in lenalidomiderefractory multiple myeloma: far from futile. Br J Haematol. 2020;188:483-5.

65. Dues DJ, Moore DJ. LRRK2 and protein aggregation in Parkinson's disease: insights from animal models. Front Neurosci. 2020;14.

66. Gracia P, Camino JD, Volpicelli-Daley L, Cremades N. Multiplicity of $\alpha$-synuclein aggregated species and their possible roles in disease. Int J Mol Sci. 2020;21.

67. Winner B, Jappelli R, Maji SK, Desplats PA, Boyer L, Aigner $\mathrm{S}$, et al. In vivo demonstration that alpha-synuclein oligomers are toxic. Proc Natl Acad Sci U S A. 2011;108:4194-9.

68. Karpinar DP, Balija MBG, Kügler S, Opazo F, Rezaei-Ghaleh $\mathrm{N}$, Wender N, et al. Pre-fibrillar alpha-synuclein variants with impaired beta-structure increase neurotoxicity in Parkinson's disease models. EMBO J. 2009;28:3256-68.

69. Majbour NK, Vaikath NN, van Dijk KD, Ardah MT, Varghese $\mathrm{S}$, Vesterager LB, et al. Oligomeric and phosphorylated alphasynuclein as potential CSF biomarkers for Parkinson's disease. Mol Neurodegener. 2016;11:7.

70. Sharon R, Bar-Joseph I, Frosch MP, Walsh DM, Hamilton JA, Selkoe DJ. The formation of highly soluble oligomers of alphasynuclein is regulated by fatty acids and enhanced in Parkinson's disease. Neuron. 2003;37:583-95. 
71. Tokuda T, Qureshi MM, Ardah MT, Varghese S, Shehab SAS, Kasai T, et al. Detection of elevated levels of $\alpha$-synuclein oligomers in CSF from patients with Parkinson disease. Neurology. 2010;75:1766-72.

72. Danzer KM, Krebs SK, Wolff M, Birk G, Hengerer B. Seeding induced by alpha-synuclein oligomers provides evidence for spreading of alpha-synuclein pathology. J Neurochem. 2009;111:192-203.

73. Hansen C, Angot E, Bergström A-L, Steiner JA, Pieri L, Paul G, et al. $\alpha$-Synuclein propagates from mouse brain to grafted dopaminergic neurons and seeds aggregation in cultured human cells. J Clin Invest. 2011;121:715-25.

74. Kordower JH, Chu Y, Hauser RA, Freeman TB, Olanow CW. Lewy body-like pathology in long-term embryonic nigral transplants in Parkinson's disease. Nat Med. 2008;14:504-6.

75. Li J-Y, Englund E, Holton JL, Soulet D, Hagell P, Lees AJ, et al. Lewy bodies in grafted neurons in subjects with Parkinson's disease suggest host-to-graft disease propagation. Nat Med. 2008;14:501-3.

76. Olanow CW, Prusiner SB. Is Parkinson's disease a prion disorder? Proc Natl Acad Sci USA. 2009;106:12571-2.

77. Emmanouilidou E, Vekrellis K. Exocytosis and spreading of normal and aberrant $\alpha$-synuclein. Brain Pathol. 2016;26:398-403.

78. Klegeris A, Pelech S, Giasson BI, Maguire J, Zhang H, McGeer EG, et al. Alpha-synuclein activates stress signaling protein kinases in THP-1 cells and microglia. Neurobiol Aging. 2008;29:739-52.

79. Lee E-J, Woo M-S, Moon P-G, Baek M-C, Choi I-Y, Kim W-K, et al. Alpha-synuclein activates microglia by inducing the expressions of matrix metalloproteinases and the subsequent activation of protease-activated receptor-1. J Immunol. 2010;185:615-23.

80. Stefanis L, Emmanouilidou E, Pantazopoulou M, Kirik D, Vekrellis K, Tofaris GK. How is alpha-synuclein cleared from the cell? J Neurochem. 2019;150:577-90.

81. Yamada K, Iwatsubo T. Extracellular $\alpha$-synuclein levels are regulated by neuronal activity. Mol Neurodegener. 2018;13:9.

82. Pistacchi M, Gioulis M, Sanson F, De Giovannini E, Filippi G, Rossetto F, et al. Gait analysis and clinical correlations in early Parkinson's disease. Funct Neurol. 2017;32:28-34.

83. Pisanu A, Lecca D, Mulas G, Wardas J, Simbula G, Spiga S, et al. Dynamic changes in pro- and anti-inflammatory cytokines in microglia after PPAR- $\gamma$ agonist neuroprotective treatment in the MPTPp mouse model of progressive Parkinson's disease. Neurobiol Dis. 2014;71:280-91.

84. Edison P, Ahmed I, Fan Z, Hinz R, Gelosa G, Ray Chaudhuri K, et al. Microglia, amyloid, and glucose metabolism in Parkinson's disease with and without dementia. Neuropsychopharmacol Off Publ Am Coll Neuropsychopharmacol. 2013;38:938-49.

85. Gerhard A, Pavese N, Hotton G, Turkheimer F, Es M, Hammers A, et al. In vivo imaging of microglial activation with [11C](R)-PK11195 PET in idiopathic Parkinson's disease. Neurobiol Dis. 2006;21:404-12.

86. Iannaccone S, Cerami C, Alessio M, Garibotto V, Panzacchi $\mathrm{A}$, Olivieri $\mathrm{S}$, et al. In vivo microglia activation in very early dementia with Lewy bodies, comparison with Parkinson's disease. Parkinsonism Relat Disord. 2013;19:47-52.

87. Ouchi Y, Yoshikawa E, Sekine Y, Futatsubashi M, Kanno T, Ogusu T, et al. Microglial activation and dopamine terminal loss in early Parkinson's disease. Ann Neurol. 2005;57:168-75.

88. Terada T, Yokokura M, Yoshikawa E, Futatsubashi M, Kono S, Konishi T, et al. Extrastriatal spreading of microglial activation in Parkinson's disease: a positron emission tomography study. Ann Nucl Med. 2016;30:579-87.

89. Chen X, Hu Y, Cao Z, Liu Q, Cheng Y. Cerebrospinal fluid inflammatory cytokine aberrations in Alzheimer's disease, Parkinson's disease and amyotrophic lateral sclerosis: a systematic review and meta-analysis. Front Immunol. 2018;9:2122.

90. Karpenko MN, Vasilishina AA, Gromova EA, Muruzheva ZM, Bernadotte A. Interleukin-1 $\beta$ interleukin-1 receptor antagonist, interleukin-6, interleukin-10, and tumor necrosis factor- $\alpha$ levels in $\mathrm{CSF}$ and serum in relation to the clinical diversity of Parkinson's disease. Cell Immunol. 2018;327:77-82.

91. López González I, Garcia-Esparcia P, Llorens F, Ferrer I. Genetic and transcriptomic profiles of inflammation in neurodegenerative diseases: Alzheimer, Parkinson, Creutzfeldt-Jakob and tauopathies. Int J Mol Sci. 2016;17:206.

92. Collins LM, Toulouse A, Connor TJ, Nolan YM. Contributions of central and systemic inflammation to the pathophysiology of Parkinson's disease. Neuropharmacology. 2012;62:2154-68.

93. Imamura K, Hishikawa N, Ono K, Suzuki H, Sawada M, Nagatsu $\mathrm{T}$, et al. Cytokine production of activated microglia and decrease in neurotrophic factors of neurons in the hippocampus of Lewy body disease brains. Acta Neuropathol. 2005;109:141-50.

94. Dursun E, Gezen-Ak D, Hanağası H, Bilgiç B, Lohmann E, Ertan $\mathrm{S}$, et al. The interleukin 1 alpha, interleukin 1 beta, interleukin 6 and alpha-2-macroglobulin serum levels in patients with early or late onset Alzheimer's disease, mild cognitive impairment or Parkinson's disease. J Neuroimmunol. 2015;283:50-7.

95. Lindqvist D, Kaufman E, Brundin L, Hall S, Surova Y, Hansson O. Non-motor symptoms in patients with Parkinson's disease - correlations with inflammatory cytokines in serum. PLoS One. 2012;7:e47387.

96. Chen J, Liu X, Zhong Y. Interleukin-17A: The key cytokine in neurodegenerative diseases. Front Aging Neurosci. 2020;12:566922.

97. Dutta D, Kundu M, Mondal S, Roy A, Ruehl S, Hall DA, et al. RANTES-induced invasion of Th17 cells into substantia nigra potentiates dopaminergic cell loss in MPTP mouse model of Parkinson's disease. Neurobiol Dis. 2019;132:104575.

98. Reale M, Greig NH, Kamal MA. Peripheral chemo-cytokine profiles in Alzheimer's and Parkinson's diseases. Mini Rev Med Chem. 2009;9:1229-41.

99. Tang P, Chong L, Li X, Liu Y, Liu P, Hou C, et al. Correlation between serum RANTES levels and the severity of Parkinson's disease. Oxid Med Cell Longev. 2014;2014:208408.

Publisher's Note Springer Nature remains neutral with regard to jurisdictional claims in published maps and institutional affiliations. 


\section{Authors and Affiliations}

Maria Francesca Palmas ${ }^{1}$ - Anna Ena ${ }^{1}$. Chiara Burgaletto ${ }^{2}$ - Maria Antonietta Casu ${ }^{3}$. Giuseppina Cantarella ${ }^{2}$. Ezio Carboni ${ }^{1}$. Michela Etzi ${ }^{1}$ - Alfonso De Simone ${ }^{4}$. Giuliana Fusco ${ }^{5}$. Maria Cristina Cardia ${ }^{6}$. Francesco Lai ${ }^{6}$. Luca Picci $^{6}$. David Tweedie ${ }^{7}$. Michael T. Scerba ${ }^{7} \cdot$ Valentina Coroneo $^{8} \cdot$ Renato Bernardini $^{2} \cdot$ Nigel H. Greig ${ }^{7}$. Augusta Pisanu ${ }^{9} \cdot$ Anna R. Carta ${ }^{1}$

1 Department of Biomedical Sciences, University of Cagliari, Cagliari, Italy

2 Department of Biomedical and Biotechnological Sciences, University of Catania, Catania, Italy

3 CNR Institute of Translational Pharmacology, Cagliari, Italy

4 Department of Pharmacy, University of Naples Federico II, Naples, Italy

5 Centre for Misfolding Diseases, Department of Chemistry, University of Cambridge, Cambridge, UK
6 Department of Life and Environmental Sciences, University of Cagliari, Cagliari, Italy

7 Drug Design \& Development Section, Translational Gerontology Branch, National Institute On Aging, National Institutes of Health, Baltimore, MD, USA

8 Department of Medical Sciences and Public Health, University of Cagliari, Cagliari, Italy

9 National Research Council, Institute of Neuroscience, Cagliari, Italy 